\title{
New frontiers in time-domain diffuse optics, a review
}

\author{
Antonio Pifferi \\ Davide Contini \\ Alberto Dalla Mora \\ Andrea Farina \\ Lorenzo Spinelli \\ Alessandro Torricelli
}




\title{
New frontiers in time-domain diffuse optics, a review
}

\author{
Antonio Pifferi, ${ }^{\mathrm{a}, \mathrm{b}}$ Davide Contini, ${ }^{\mathrm{a}}$ Alberto Dalla Mora, ${ }^{\mathrm{a}}$ Andrea Farina, ${ }^{\mathrm{b}}$ Lorenzo Spinelli, ${ }^{\mathrm{b}}$ and \\ Alessandro Torricelli ${ }^{a, b, *}$ \\ aPolitecnico di Milano, Dipartimento di Fisica, Piazza Leonardo da Vinci 32, Milan I-20133, Italy

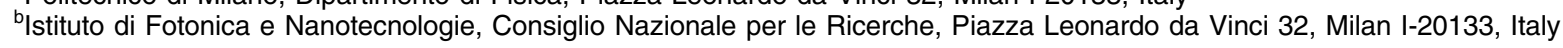

\begin{abstract}
The recent developments in time-domain diffuse optics that rely on physical concepts (e.g., timegating and null distance) and advanced photonic components (e.g., vertical cavity source-emitting laser as light sources, single photon avalanche diode, and silicon photomultipliers as detectors, fast-gating circuits, and time-to-digital converters for acquisition) are focused. This study shows how these tools could lead on one hand to compact and wearable time-domain devices for point-of-care diagnostics down to the consumer level and on the other hand to powerful systems with exceptional depth penetration and sensitivity. $\odot$ The Authors. Published by SPIE under a Creative Commons Attribution 3.0 Unported License. Distribution or reproduction of this work in whole or in part requires full attribution of the original publication, including its DOI. [DOI: 10.1117/1.JBO.21.9.091310]
\end{abstract}

Keywords: biomedical optics; absorption; scattering; reflectance; photonics; picosecond phenomena.

Paper 160063SSVRR received Jan. 30, 2016; accepted for publication May 24, 2016; published online Jun. 17, 2016.

\section{Introduction}

The study of photon propagation in highly scattering media (diffuse optics) is a powerful tool to noninvasively investigate biological tissues in depth. ${ }^{1,2}$ For most biological media in the 600 - to $1100-\mathrm{nm}$ range, the scattering coefficient is much higher than the absorption coefficient, thus hindering information from the human body that in absence of scattering would appear transparent as a feebly colored glass. The attractiveness of probing the human tissues and their functions with light has fostered great efforts to cope with the challenges of "diffuse optics," leading to a whole range of new physical concepts, theoretical models, instruments, measurement approaches, and applications. The terms near-infrared spectroscopy (NIRS) and diffuse optical imaging (DOI) are used in the scientific community to refer to the techniques used for noninvasive monitoring and imaging with diffuse light. Typically, light is injected into the medium and the signal re-emitted at some distance(s) on the surface is analyzed to retrieve information carried by photons along their paths. In the simplest case, continuous wave $(\mathrm{CW})$ illumination and detection are employed. ${ }^{3-5}$ Conversely, the adoption either of a frequency-domain (FD) ${ }^{6}$ or of a time-domain (TD) $)^{7-10}$ scheme permits to add a further dimension in the measurement, e.g., disentangling absorption from scattering contributions.

This review is focused on the specific advantages and perspectives of the TD approach, whereas a more comprehensive comparison among the different modalities can be found elsewhere. ${ }^{1,11,12}$ On the occasion of the 20th anniversary of the foundation of the Journal of Biomedical Optics - the first journal to focus specific interest around the diffuse optics applications and community-it is time to take stock and try to develop a long-term vision on the perspectives in the field.

The first proposals of TD diffuse optics applied to biological media date back to the late 1980s. ${ }^{13-16}$ Since then, the technology has advanced through major steps, which in turn brought about new applications. The first generation of TD systems was based on large table-top pulsed lasers combined with expensive

${ }^{\star}$ Address all correspondence to: Alessandro Torricelli, E-mail: alessandro torricelli@polimi.it streak-camera or a delicate microchannel-plate (MCP) photomultiplier (PMT) coupled to rack-hosted nuclear instrumentation module (NIM) for time-correlated single-photon counting (TCSPC). ${ }^{17-21}$ These laboratory systems permitted to explore the fundamentals of diffuse optics in the $\mathrm{TD}^{18,20,22-24}$ and to obtain the first basic measurements on the optical characterization of biological tissues in vivo. ${ }^{17,25-27}$

The second generation was made possible by the introduction of compact and rugged light sources, detectors, and electronics. ${ }^{28,29}$ In particular, sources were provided by pulsed diode lasers, while for detection metal-channel dynodes PMTs were proposed. Processing electronics were provided by TCSPC boards hosted in a personal computer. These components made it possible to construct rack-based systems suitable for use in clinical environments. Also, they opened the way to the seminal work on multichannel acquisition, as a key requisite for realtime imaging and tomography. ${ }^{30-33}$ Several problems that had to be faced, like dynamic signal attenuation, multipoint, multiwavelength discrimination, and sharing of the maximum countrate among multiple channels are also key issues for future high-throughput systems.

A third generation, which constitutes the actual state-of-theart, takes advantage also of fiber-based source (complemented with photonic crystal fibers for broad spectral coverage), hybrid detector, or solid-state detector [e.g., single photon avalanche diode (SPAD)], and time-to-digital converters (TDCs). ${ }^{34}$

Thus, a number of in vivo applications and clinical studies were possible with TD systems in different areas, primarily optical mammography, ${ }^{35}$ brain mapping, ${ }^{36}$ and muscle monitoring. ${ }^{37}$ In these studies, the target was mainly oxygenated and deoxygenated blood content in biological tissues. In other pilot studies, the noninvasive assessment of main tissue components like lipid, ${ }^{38}$ bone,${ }^{39}$ and collagene ${ }^{40}$ was investigated. The TD technique has been also used for the nondestructive optical characterization of food, ${ }^{41}$ wood, ${ }^{42}$ pharmaceuticals, ${ }^{43-45}$ and semiconductor powders. ${ }^{46}$

At present, the majority of TD systems are custom-made laboratory prototypes ${ }^{36,37}$ while, to our knowledge, only two complete TD diffuse optics systems are available on the market (TRS-20, Hamamatsu Ltd., Japan ${ }^{47}$ and PICOXEA TCSPC 
Analyser, Aurea Technologies, France ${ }^{48}$ ). The high costs of components, their problems of scalability, and the intrinsic higher complexity of a TD system hampered the spread of this technique.

It is worth noting that TD NIRS has been implemented also with measurement schemes different than the traditional one based on pulsed lasers and TCSPC devices. Formerly, the equivalent of the amplitude modulation was proposed for TD instruments by means of a pseudo coding modulation. ${ }^{49,50}$ Very recently, Borycki et al. ${ }^{51}$ presented an approach to TD NIRS based on the analysis of spectral interference fringe pattern as measured by a Mach-Zehnder interferometer with a frequency swept narrow linewidth laser (iNIRS). In that work, photon time-of-flight measurements have been demonstrated in a homogeneous medium (a nonabsorbing 1-cm thick glass cuvette filled with a water solution of Intralipid). Although the presented iNIRS setup is not suitable for imaging applications, the iNIRS results are promising, especially for the possibility to simultaneously record the dynamical properties of turbid media (e.g., equivalent to diffuse correlation spectroscopy). However, further work is needed to verify the performances of the iNIRS approach in realistic conditions for biomedical applications (e.g., reflectance measurements with large source-detector separation on tissue phantom with absorbing properties closer to those of in vivo biological tissue).

At present, we are just at the dawn of a next generation (nextgen) of TD systems, with a breakthrough in terms of performance, size, cost, and flexibility, which will possibly result in great impact on new and widespread applications. This breakthrough is enabled by two factors: on one side, the physics of TD diffuse optics, which offers unique properties to boost sensitivity, resolution, and quantitation, and on the other side, the enormous recent advancements of photonics, which enables a new class of optoelectronics components and devices.

We have tried to render our vision of the diffuse optics field by means of a modified hype cycle diagram. ${ }^{52}$ In Fig. 1, we have illustrated our expectations in terms of visibility, usability, and relative maturity for the CW approach and of the TD approach. For the latter case, we have distinguished between the classic TD systems, including up to the third generation of TD systems, and the next-gen TD systems. In the last 20 years, the CW

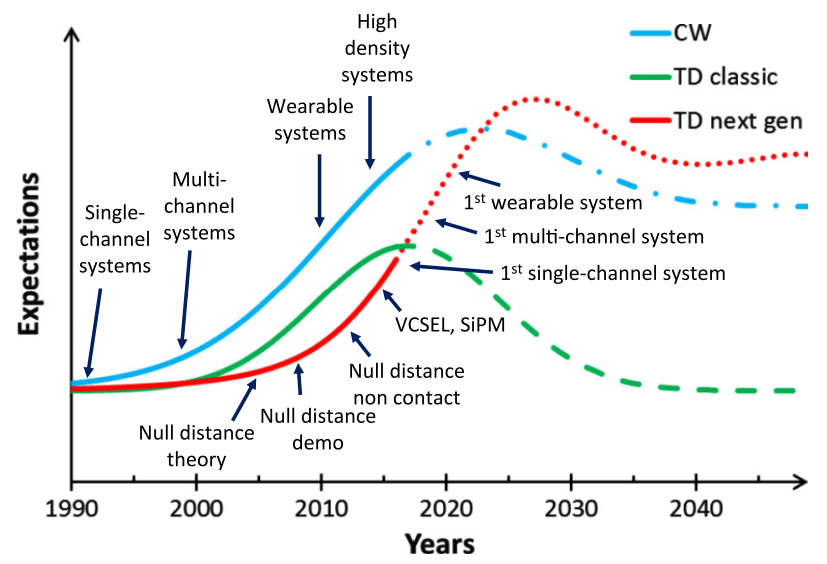

Fig. 1 Modified hype cycle diagram for the CW approach, the classic TD approach, and for the next-gen TD approach. The diagram represents the authors' interpretation of the hype cycle of these technologies. approach has been characterized by a strong rise in expectations. Indeed, the CW approach is at the moment very strong, with clinically accepted fields of application and very strong research related to high-density NIRS. ${ }^{53}$ However, the noticeable advances in technology, which have allowed the development of wearable devices or of complex multichannel and multiwavelength instruments, have always been based on physical models that rely on the measurement of light attenuation, like in the very first example of application of NIRS technology. ${ }^{54}$ We believe that only with a synergic approach between physics and technology, will it be possible for the diffuse optics field to sustain its growth. This is what is now happening in the next-gen TD approach.

The structure of the paper aims to present the foundations stemming from recent research at the basis of the next-gen diffuse optics and to prefigure the most promising outcomes in terms of applications. More specifically, the physics of TD diffuse optics is presented in Sec. 2, the technological advancements toward next-gen devices are discussed in Sec. 3, while the long-term vision and future applications are highlighted in Sec. 4.

\section{Physical Concepts}

In this section, we briefly outline the main physical concepts that are peculiar to the TD approach. A comprehensive description of the physics of photon migration in the framework provided by the radiative transport theory and the diffusion theory can be found in Ref. 55.

In the wavelength range between 600 and $1100 \mathrm{~nm}$, two main phenomena affect the photon trajectories in biological tissues: scattering and absorption. When a scattering event occurs, the photon is deviated. Scattering is caused by microscopic refractive index changes inside the tissue, which are linked to its structural properties. ${ }^{56}$ On the other hand, the absorption phenomenon, with the consequent annihilation of a photon, is due to the presence of particular chromophores inside the tissue that convert light intensity into other kinds of energy, either radiative (i.e., fluorescence) or nonradiative (i.e., vibrational). Both the scattering and the absorption interactions can be described by means of simple coefficients, $\mu_{\mathrm{s}}^{\prime}$ and $\mu_{\mathrm{a}}$, respectively, which

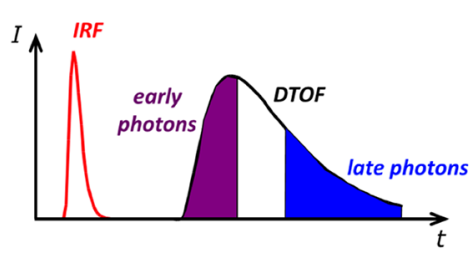

(a)

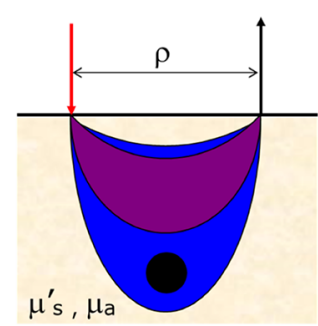

(b)
Fig. 2 (a) Scheme of a time-resolved NIRS measurement in reflectance geometry: the injected light pulse [in terms of the instrument response function (IRF), red] and the broadened DTOF (black), detected at a distance $\rho$ from the injected point, are represented as a function of time. Time-gating of the DTOF allows a selective probing of the diffuse medium: photons with early (magenta) and late (blue) arrival times probe different regions of the diffusive medium (magenta and blue areas, respectively, in panel b). In particular, only late photons are sensitive to the localized black inclusion deeply embedded in the diffusive medium. 
represent the inverse of the free-mean path between consecutive scattering and absorption events.

In the TD approach, a pulse of light with the duration of tens of picoseconds is injected into the tissue, typically exploiting a suitable optical fiber. The light pulse then propagates inside the tissue and it can be eventually detected at a certain distance from the injection point (typically in the range from 10 to $40 \mathrm{~mm}$ ) [see Fig. 2(a)].

Besides the reflectance configuration described above, it is also possible to investigate biological tissues by means of a transmittance approach, where source and detector fibers are located in opposite sides of the tissue. This approach is feasible when the thickness of the tissue does not exceed values of the order of 60 to $80 \mathrm{~mm}$, and it has been successfully applied in the measurements of breast, ${ }^{57}$ calcaneus bone, ${ }^{39}$ or finger. ${ }^{58}$ Moreover, a fully tomographic approach has been applied for studying the infant brain. ${ }^{59,60}$ However, other interesting biological tissues and structures, such as heart, lung, and adult brain, are presently accessible only by the reflectance approach. Furthermore, when the transmittance approach is feasible, also the reflectance configuration can be usefully adopted.

The histogram of the time-of-flight of photons emerging from the tissue, known as the photon distribution of time-offlight (DTOF) or the temporal point spread function (TPSF), results delayed, broadened, and attenuated with respect to the injected light pulse, because of the propagation inside the diffusive medium. In particular, the delay is due to the finite time that light takes to travel the distance between the injection and detection point; the broadening is mainly due to the different paths that photons travel because of multiple scattering. The attenuation appears because both absorption and scattering phenomena remove photons from the considered direction. Increasing the scattering yields an increased delay and broadening of the DTOF and decreases the number of detected photons.
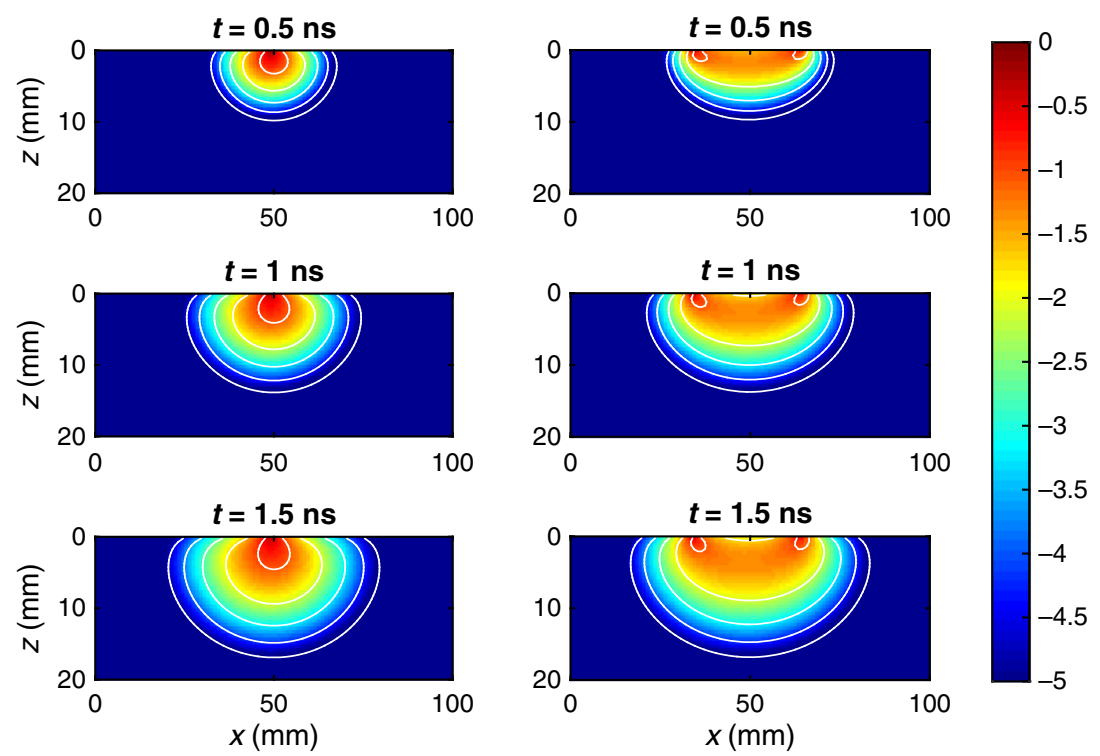

(b)

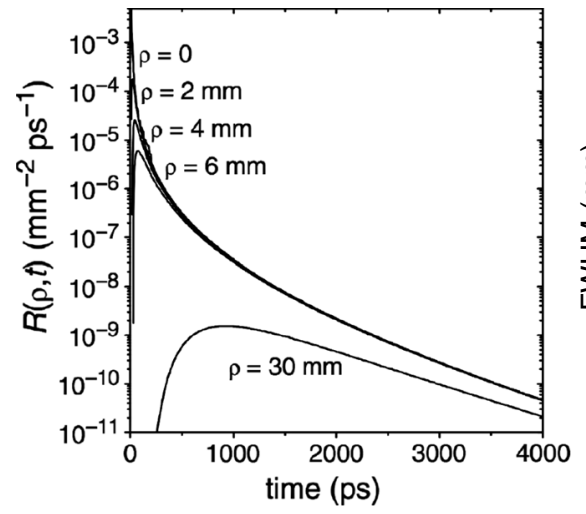

(c)

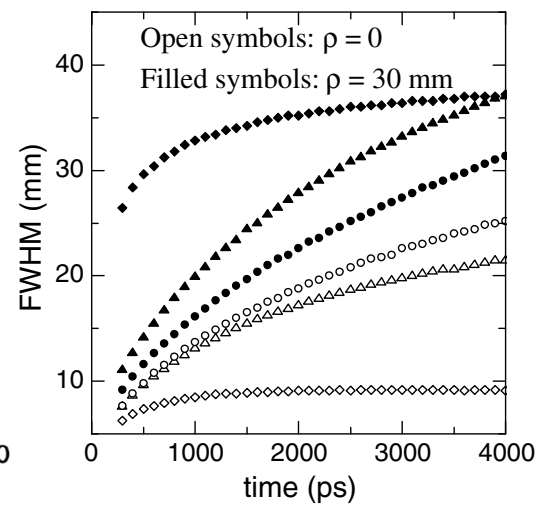

(d)

Fig. 3 Sensitivity profiles $\left(\log _{10}\right)$ for $(\mathrm{a}) \rho=0$ and (b) for $\rho=30 \mathrm{~mm}$ in a homogeneous diffusive medium at three times $(0.5,1,1.5 \mathrm{~ns})$. Each white line represents the contour edge of the contrast at every decade. (c) TPSF for small source-detector separations $\rho$ and for $\rho=30 \mathrm{~mm}$ in a homogeneous diffusive medium. Data for $t<500 \mathrm{ps}$ and $\rho<5 \mathrm{~mm}$ were obtained by Monte Carlo simulations, while the diffusion equation was used for longer times and larger distances. (d) Full width at half maximum (FWHM) of the contrast line scans for $\rho=0$ (open symbols) and for $\rho=30 \mathrm{~mm}$ (filled symbols) for different depths ( $5 \mathrm{~mm}$, diamond; $15 \mathrm{~mm}$, triangle; $25 \mathrm{~mm}$, circle) of an absorption perturbation [Figs. 3(c) and 3(d) have been taken from Ref. 68]. 
Finally, absorption affects both the signal intensity and the trailing edge (i.e., slope of the tail) of the DTOF, leaving the temporal position of the DTOF substantially unchanged.

Because scattering and absorption phenomena affect differently the DTOF, for homogeneous media, $\mu_{\mathrm{s}}^{\prime}$ and $\mu_{\mathrm{a}}$ can be obtained exploiting a single-distance TD measurement. This aspect, grounded on the physics of photon migration in diffusive media, is one of the peculiarities of the TD approach that differentiates it from the other approaches.

As a matter of fact, in many cases, the biological tissues of interest are well below the skin surface: the brain cortex and the muscle are usually studied, but also the lung or the heart could be possible targets. Then, the penetration capability of diffuse light is crucial for this extent. In the $\mathrm{CW}$ approach, the only way to increase the average penetration depth of photons inside a diffusive medium is to increase the distance between the injection and detection points. ${ }^{61}$ Proceeding in this way, however, has several drawbacks. First, the explored volume by photons is very large, with the consequence of a reduced lateral spatial resolution and a reduced sensitivity, in terms of fraction of detected photons that have effectively visited the region of interest. Second, the increase of the source-detector distance unavoidably causes a reduction of detected photons and, then, of the signal-to-noise ratio (SNR) of the measure. Finally, the penetration depth strongly depends on the absorption coefficient, ${ }^{61}$ resulting in different probing depth at different wavelengths.

In the TD approach, instead, the average depth reached by a photon is directly related to the average time spent inside the tissue, before being eventually detected: the larger the arrival time of photons with respect to the injection time, the deeper the photons penetrate inside the tissue. ${ }^{61,62}$ Obviously, it is also true that the larger the arrival time, the lower the number of emerging photons, with negative effects on the SNR, but, in principle, the information on depth structures is physically available. Then, in the TD approach, the information on the reached depth is encoded in the arrival time of the photons, with marginal dependence on the source-detector distance. This fact is a peculiarity of the TD approach and gives incomparable advantages, with respect to the other techniques, that can be exploited. For instance, the sensitivity to deep structures can be enhanced by a time-gating operation to select only the late arrival photons in the DTOF [see Fig. 2(b)]. ${ }^{63}$ Moreover, by considering a more complex time-slicing of the DTOF, it is possible to uncouple in the measured signal contributions coming from deeper structures from those due to shallower ones. ${ }^{64-67}$

Another inherent advantage exhibited by the TD approach is that, since the photon penetration capability does not depend on source-detector distance, measurements at null source-detector distance also are conceivable. This possibility is important, because the lateral extension of the diffusive medium explored by photons is reduced to the minimum, and the lateral resolution reaches its physical limit [see Figs. 3(a) and 3(b)].

In two pioneering papers, ${ }^{68,69}$ the feasibility of the null distance approach and its advantages in terms of optimal signal level, contrast, and spatial resolution were demonstrated both theoretically and experimentally. However, this approach is achievable only by applying an efficient time-gating mechanism that extracts the few, useful, late (deep travelling) photons out of the overwhelming burst of early (superficial) photons. Indeed, the number of early arriving photons becomes increasingly

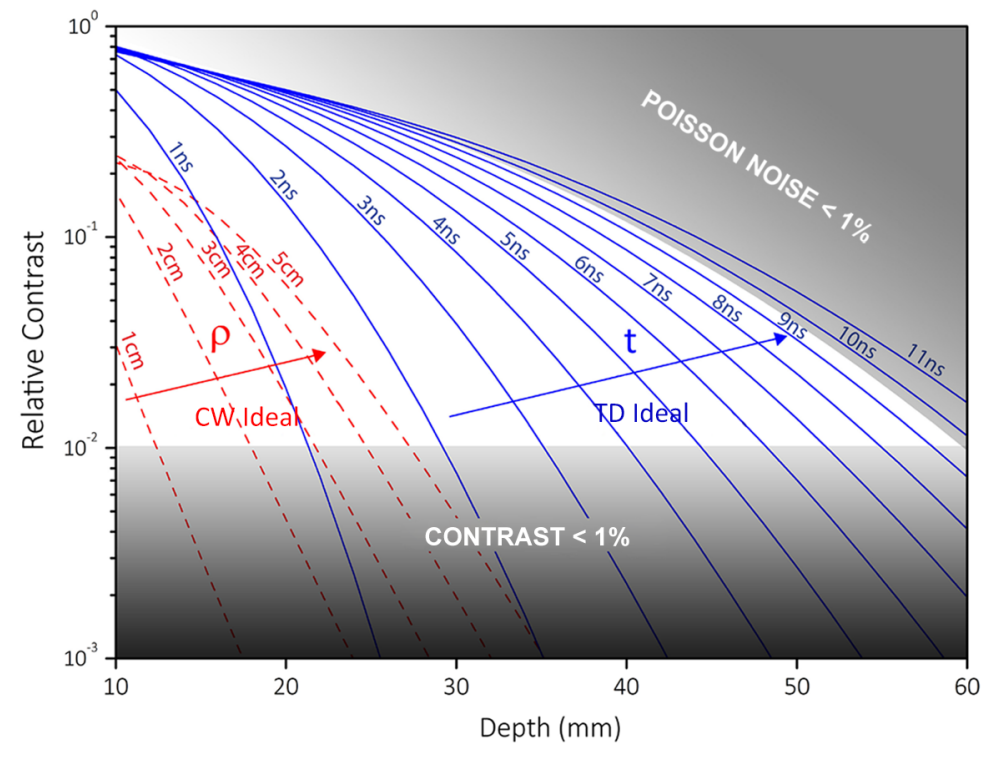

(a)

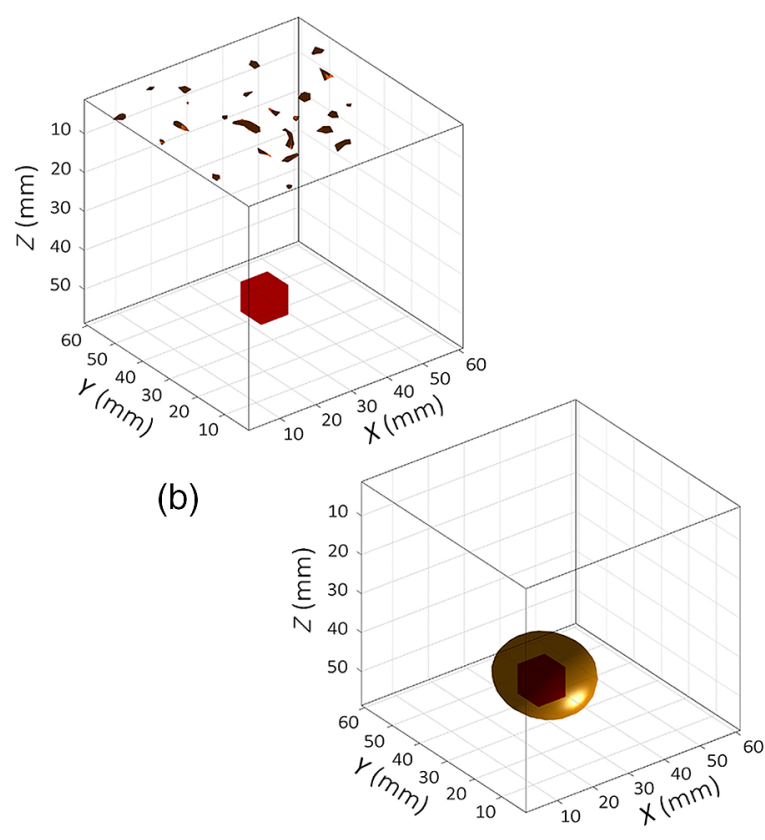

(c)

Fig. 4 (a) Simulations of the contrast produced by an absorption perturbation set at increasing depths $z$ in a homogeneous diffusive medium $\left(\mu_{\mathrm{a}}=0.1 \mathrm{~cm}^{-1}, \mu_{\mathrm{s}}^{\prime}=10 \mathrm{~cm}^{-1}\right)$ and related to an ideal TD (blue solid lines) or CW (red dashed lines) system. The detectability region is represented in white. TD data are obtained at $\rho=0$ at increasing delays. CW data are obtained at increasing source-detector distances. Reconstruction results of the tomography performed using (b) CW and (c) TD simulated data. The represented surfaces correspond to the half of the maximum of the absorption change $\Delta \mu_{\mathrm{a}}$ obtained in all the volume. The simulated perturbation is also depicted in red as reference (this figure has been taken from Ref. 73). 
larger, relatively to the late arriving photons, when the sourcedetector separation becomes smaller and smaller [see Fig. 3(c)]. Exploiting a fast-gated detector, the null source-detector distance approach has been successfully applied at a laboratory level for noninvasive detection of brain activity using a single optode, ${ }^{69}$ single-fiber interstitial optical biopsy, ${ }^{70}$ noncontact deep tissue functional imaging, ${ }^{71}$ and diffuse optical tomography (DOT) using a compact probe with few injection-collection fibers. $^{72}$

The adoption of the null source-detector distance approach permits to investigate the ultimate physical limits of TD diffuse optics in terms of spatial resolution, sensitivity, and depth penetration under the maximum safe laser exposure. In Fig. 4(a), it is reported that the contrast produced by a localized absorption perturbation set at increasing depths $z$ in a homogeneous diffusive medium, when densely packed ideal sources and detectors (100\% fill factor on $1-\mathrm{cm}^{2}$ optode, unitary quantum efficiency, perfect gating capability) are considered, with laser exposure and acquisition time compatible with clinical environment. ${ }^{73}$ In the CW approach, depth sensitivity is limited by the maximal usable source-detector distance, whereas the ideal TD system can explore deep structures even at null distance provided that enough late photons are collected. In particular, a localized absorption perturbation of $0.1 \mathrm{~cm}^{-1}$ over a $1-\mathrm{cm}^{3}$ volume can be potentially detected down to a maximal depth of $6 \mathrm{~cm}$, which represents the physical limit of detectability, assuming as detectability criteria a contrast $>1 \%$, with a Poisson noise level $<1 \%$ (i.e., with a minimum of $10^{4}$ detected photons). We note that the physical limit is set by assuming the maximum possible photon harvesting. In an ideal TD system, this is guaranteed by a dense source and detector distribution on the optode, a configuration reliable only because of the null distance approach. The ideal TD scheme is optimal also for three-dimensional (3-D) tomography in reflectance geometry, as shown for instance in Figs. 4(b) and 4(c).

The issue of spatial resolution (both lateral and in-depth) is surely a crucial one in DOI and tomography. While the null source-detector approach can increase spatial resolution as compared to the large source-detector separation, ${ }^{68}$ still upon increasing the photon arrival time, the spread of the photon spatial distribution increases as well resulting in a dramatic blurring of the contrast profile. As a quick example, referring to the case of Fig. 4, a point-like optical inhomogeneity set at a depth $z=$ $2.5 \mathrm{~cm}$ will produce a large relative contrast $(20 \%)$ at $t=4 \mathrm{~ns}$, but with a lateral broadening of $2.5 \mathrm{~cm}$ (FWHM), as shown in Fig. 3(d). With a proper SNR, 3-D tomographic reconstruction can increase the spatial resolution as compared to the purely planar imaging case. In the $\mathrm{CW}$ case, a dense arrangement of sources and detectors with a large SNR was proven both theoretically $^{74}$ and experimentally ${ }^{75}$ to significantly increase spatial resolution in DOT. The gain in depth sensitivity offered by the time-gated approach could also lead to better spatial resolution in DOT. Still, the question is open, and it is not settled whether a very deep inclusion with a detectable contrast could be imaged with an acceptable spatial resolution.

\section{Photonic Components}

In order to fully exploit the potentialities of the TD approach described in Sec. 2, it is fundamental to overcome the technological limitations of the components and devices currently used in TD systems.
As a general consideration, in Fig. 5, we have highlighted the evolution of TD systems in terms of volume, cost, and performances (as summed up in the simple parameter photon countper-second, cps) during the last 20 years and the foreseen development in the next 20 years to come. In the last two decades, we have experienced a reduction of about 4 orders of magnitude in the cost of detectors (from $10^{6} \$$ for a streak camera-based system to $10^{2} \$$ for a solid-state device), and a parallel increase of a similar factor in the performances (from $10^{4} \mathrm{cps}$ of NIM modules to $10^{8} \mathrm{cps}$ of the new microelectronic components).
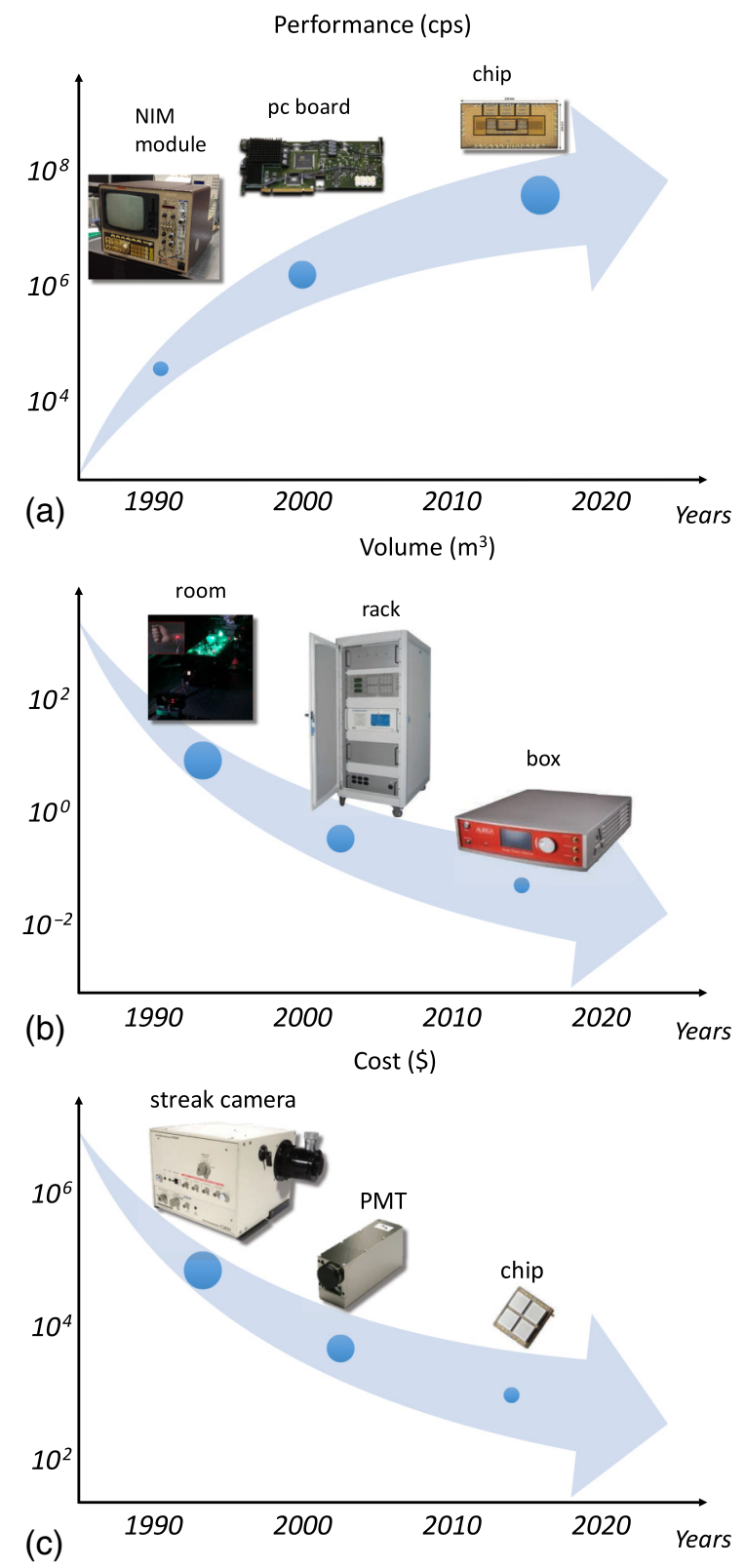

Fig. 5 Evolution of TD systems. (a) Evolution of the performances in term of photon counts-per-second. The performances have been improved of about 4 orders of magnitude from NIM module based instrumentation to new microelectronic components. (b) Evolution in term of volume. Volume has been decreased of 4 orders of magnitude from laboratory systems to portable systems. (c) Evolution in term of cost. The cost has been decreased of 4 orders of magnitude from streak-camera based systems to solid-state device based systems. 
Curiously, also the volume of a TD system has shrunk by 4 orders of magnitude (from $10^{2} \mathrm{~m}^{3}$ of a laboratory room to $10^{-2} \mathrm{~m}^{3}$ of a portable bench-top system).

In this section, we review the main building blocks of a TD system (i.e., light sources, fiber optics and accessories, detectors, and timing electronics) with a special emphasis on the future developments fostered by the incessant advance of photonic technologies.

\subsection{Light Sources}

The main characteristics of a laser source suitable for TD diffuse optics measurements are emission wavelengths in the red and near-infrared (NIR) region, where the main tissue constituents show a measurable absorption, but not as high as in the visible and infrared spectral region so as to probe tissue in depth; fullwidth at half maximum (FWHM) as narrower as possible, ideally like a delta function, so as not to hamper the time resolution of the overall system; high repetition rate $(>20 \mathrm{MHz})$ in order to allow the reconstruction of the DTOF with acquisition time compatible with in vivo imaging (around $1 \mathrm{~s}$ or less); and a sufficient average power $(>1 \mathrm{~mW})$ in order to achieve a suitable SNR. Different types of lasers fulfil these conditions starting from table-top laser systems down to compact original equipment manufacturer solutions (see Ref. 36 for a recent review).

Often in diffuse optics instruments, different wavelengths are injected in many points on the sample surface in order to perform a mapping (either tomography or topography) of the optical properties of the medium. In CW and FD systems, many wavelengths can be injected together in different points at the same time due to an amplitude modulation scheme, which guarantees the discrimination of the provenience of the detected signal. $^{76}$

A spectrally broadband light can be also injected at one point. The wavelength multiplexing, in this case, relies on the detection stage using dispersive elements like diffraction gratings..$^{19,77,78}$ It is worth noting that, by using this scheme, the injected power is fixed for the broadband light source; thus, due to spectral response of the tissue, the detected signal can strongly change among the spectrum giving strong variations in the SNR ratio among the detected wavelengths. Alternatively, spectral measurements can be performed sequentially, using a tunable filter and a proper adaptation of the injected power to the tissue attenuation. ${ }^{45}$

In TD instruments used for DOI, two are the most used injection schemes. The first one is time multiplexing, ${ }^{36}$ where pulses at different wavelengths are injected in the same point few nanoseconds one after the other. Thus, detection points in the same area of the sample collect photons of different wavelengths in the same time slot. The same scheme is sequentially repeated in all the desired points on the sample. The second scheme is space multiplexing. ${ }^{79}$ In this scheme, different wavelengths are injected in different parts of the sample enough faraway one from the other in order to avoid crosstalk. Thus, detection points in the same part of the sample collect photons of only one wavelength, while in other points, faraway from the previous ones, another wavelength is collected in the same time slot. It is necessary to switch wavelengths between the different areas of the sample in order to acquire all the wavelengths in all the points of the sample.

A tradeoff between the number of measurement points and the measuring time must be considered whichever multiplexing methods are employed. In fact, all the schemes proposed in the previous paragraphs do not contemplate light injection and detection from more than two couples of optodes at the same time. It is straightforward that a high number of measurement points need longer lighting sequences of the injection points, reducing the integration time for the photons collection in the detection points and consequently, the achievable SNR. Stateof-the-art TD instruments are able to acquire images of up to 30 points with a frequency rate of $1 \mathrm{~Hz} \cdot{ }^{80-82}$ It is worth noting that actual TD imagers are characterized by about 1 order of magnitude less laser power and detection efficiency with respect to the novel technologies presented in this paper. Another important aspect to consider is that TD instruments need less measurement points to cover the same area with respect to $\mathrm{CW}$ and FD instruments. In fact, it is not necessary to consider the use of short-distance measurement to uncouple the effect of superficial layer, because photons average penetration depth is independent of source-detector points distance. Thus, by employing the multiplexing schemes already adopted in TD instruments, it is possible to design a TD instrument with a dense arrangement of sources and detectors with an imaging rate like that of $\mathrm{CW}$ and $\mathrm{FD}$ instruments (which exploit heterodyne modulation and demodulation schemes).

The first TD instruments were based on bulky lasers as Ti: sapphire lasers. ${ }^{19,83}$ Their main characteristics are high average power (up to $1 \mathrm{~W}$ ), wide wavelength tunability (over $400 \mathrm{~nm}$ ), FWHM $<1$ ps, and repetition frequency up to $100 \mathrm{MHz}$. In particular, the large amount of available power would easily permit the multiplexing of the injection points. This choice for a laser source is suitable for a laboratory system, where the bulkiness of the laser source and the long time for wavelength swapping are not a limitation; certainly, this is not the proper solution for a system designed for in vivo measurements in a clinical environment.

In recent years, different pulsed fiber lasers based on supercontinuum generation appeared on the market, ${ }^{84,85}$ with typical total average power of 5 to $10 \mathrm{~W}$ spread over a large spectrum (400 to $2000 \mathrm{~nm}$ ), pulses with FWHM $<10 \mathrm{ps,} \mathrm{and} \mathrm{repetition}$ frequency of tens of megahertz. These laser sources, unlike the Ti:sapphire lasers, are compact and suitable for a portable system, ${ }^{86,87}$ and also wavelength selection can be performed more rapidly. These characteristics fostered the widening of the application of diffuse optics beyond the classical therapeutic window (600 to $1000 \mathrm{~nm}$ ). The main drawbacks of this technology remain the high cost, the difficult power stabilization for the nonlinear effects in the supercontinuum generation, and in general, the limited robustness of the laser system.

The most commonly employed light sources in TD diffuse optics instruments are pulsed diode lasers. ${ }^{80-82,88}$ They are edge emitting laser diodes operated in gain switching mode in order to produce a train of pulses with FWHM less than 100 to 200 ps, average power of some milliwatts, and repetition rate up to $100 \mathrm{MHz}$. The costs and compactness of these sources are sufficient for use in portable systems but not enough to think to a multiplication of the laser units (e.g., more than 8) in the same instrument. The main limitation of this kind of laser is the strict correlation between laser average power and FWHM of the pulses, which is intrinsically dependent on the gain switching operation mode. In fact, it is hard to increase the average power over a few milliwatts without broadening the laser pulses. A recent development of these lasers is the miniaturization and rationalization of the driving electronics tailoring the laser source to the application and transforming the laser head into 
a stand-alone object. ${ }^{89,90}$ This type of light source is going to be used in a compact and hybrid (integrating TD diffuse optics and diffusion correlation spectroscopy) medical device for brain monitoring in premature babies. ${ }^{91}$ All the laser sources discussed, independently of the technology exploited, cannot be directly integrated on the probe in contact with the tissue but can require a guiding optics to deliver light pulses to the sample.

In the last year, a first demonstration of a TD diffuse optics instrument with the pulsed laser source directly integrated into the probe was presented. ${ }^{73}$ The probe was based on a vertical cavity source-emitting laser (VCSEL) with custom-made pulsing electronics. In this work, the source, directly in contact with the tissue, was able to emit around of $1 \mathrm{~mW}$ of average power, with a repetition frequency of $40 \mathrm{MHz}$ and a FWHM of $250 \mathrm{ps}$. The fact that in VCSEL lasers, the cavity is grown vertically and not horizontally as in the edge-emitting lasers, reduces productions costs, ensures a higher aperture, and thus, a lower divergent beam, and reduces power consumption even if the average power emission in VCSEL is lower than in other semiconductor lasers. Furthermore, the planar geometry permits to design and build up two-dimensional arrays of VCSELs. The compactness of this source, given by the fact that the laser cavity and the ancillary circuitry can be hosted into a single application-specific integrated circuit (ASIC), and its low cost opens the possibility to have tens of laser sources directly on the probe, without the necessity to multiplex the laser power of a single source between different injection points. This possibility can lead to a dense arrangement of measurement points, thus improving the achievable SNR, and consequently reducing the acquisition times and obviously shrinking the overall dimensions of the system, in particular, for multichannel instruments. This kind of scenario will surely need to cope with power dissipation issues. Indeed, while single-photon detectors and timing electronics already started to be integrated into a single lowpower chip without the need of additional ancillary electronics (see Secs. 3.3 and 3.4), the present implementation of TD system with a probe-hosted laser source ${ }^{73}$ is still based on a discrete component electronics design, which typically involves higher power consumption to obtain the intense and fast current transitions needed for picosecond pulsing. It is thus required to develop both high-efficiency ASIC solutions to replace discrete components and proper heat management strategies.

The most important drawbacks for the use in TD diffuse optics are the limited availability of wavelengths on the market, with respect to other laser technologies, and the relatively low emitted power. This last issue can be overcome by pulsing an array of VCSELs instead of a single chip. Nowadays, many application fields, where shorter wavelengths in the visible and NIR region are becoming of interest, can be envisaged for VCSELs: biomedical optics, sensing, consumer electronics, and so on. ${ }^{92}$ The increased interest in this technology will push the development of VCSELs emitting in a spectral region suitable for diffuse optics.

\subsection{Fiber Optics and Accessories}

A proper optical system is needed in order to deliver light pulses onto the sample and to collect re-emitted light from it. In the TD approach, both injection and detection optics should not broaden light pulses and should guarantee the maximum coupling efficiency between the instrument and sample.

TD systems typically use multimode single fibers or fiber bundles directly in contact with the tissue to deliver light.
Optical fibers can be characterized in terms of few parameters relevant for the application in diffuse optics: refractive index profile (graded index or step index), numerical aperture (NA), diameter, and attenuation. Furthermore, for in vivo application, robustness, rigidity, and other mechanical aspects must also be considered.

Graded index fibers typically show negligible temporal dispersion $(<1 \mathrm{ps} / \mathrm{m})$ but have lower core diameter $(<200 \mu \mathrm{m})$ and NA $(<0.3)$ compared to step index fibers, which have a high core diameter (up to $3 \mathrm{~mm}$ ) and NA $(<0.6)$, but also a higher temporal dispersion $(<100 \mathrm{ps} / \mathrm{m})$. In terms of attenuation, in the range of wavelengths of interest for diffuse optics, we can identify two categories: glass fibers with lower attenuation $(<0.001 \mathrm{~dB} / \mathrm{m})$ and plastic fibers with higher attenuation $(<0.2 \mathrm{~dB} / \mathrm{m})$. The type of material also implies the mechanical characteristics: in fact, glass fibers are more rigid and heavier than plastic fibers for the same core diameter.

Injection fibers do not need a high core diameter or high NA, because it is quite easy to focus a collimated laser beam also in a small core fiber; for this reason, the best choice for injection optics is to use a graded index glass fiber (with 62.5- or 100- $\mu \mathrm{m}$ core diameter) in order to prevent losses and pulse broadening.

Conversely, when the optical etendue ${ }^{93}$ of the detector is much higher than the one of the detection fibers, detection fibers need a large core and large NA in order to maximize light harvesting. Unfortunately, large core glass fibers are too rigid to be practically used in TD instruments for in vivo measurements, while plastic fibers show higher attenuation. For these reasons, bundles of small-core glass step index fibers, with a high ratio between the core and cladding diameter to optimize the filling factor, are normally used; bundles of graded index fibers are usually discarded because of their low filling factor resulting in an insufficient active area. Step index fiber bundles maximize light harvesting but increase the temporal broadening. ${ }^{94}$ Recently, large-core plastic graded index fibers appeared on the market that were able to show negligible pulse broadening $(<1 \mathrm{ps} / \mathrm{m})$ but introduced very high losses $(<0.2 \mathrm{~dB} / \mathrm{m})$, which limit the usable length of these fibers to a few meters. ${ }^{95,96}$

A new fascinating perspective is the adoption of a noncontact scheme, where light is injected to and collected from the tissue using distant remote optics. In this case, the broadening of the pulse is not an issue because most of laser light propagation happens in free space, but the maximization of light harvesting and the reduction of reflection remain the main problems. The noncontact approach, now at a very early stage, is very attractive for numerous applications ranging from neonatal monitoring to wounds studies. In Refs. 71 and 97, an example of a noncontact imager was presented, where a dense geometry of measurement points was obtained, employing a galvanometric mirror in order to scan the sample surface. The detection and acquisition scheme were based on the null distance approach with timegated electronics, with the added value to suppress direct reflections at the detector originating from the noncontact scheme.

Each implemented solution represents a tradeoff between collection efficiency and pulse broadening. ${ }^{98}$ The optimum solution should avoid fibers or bulk optics and, when the application allows to touch the sample, it should place sources and detectors directly in contact with the sample. Indeed, as we described in Sec. 3.1, it is possible to integrate pulsed laser sources directly into the probe. In the following Secs. 3.3 and 3.4 , we present solutions in order to integrate into the probe also the detectors and the timing electronics. The recent 
developments of photonics and optics can open wide scenarios of applications in the diffuse optics field. One possible application could be the microlens arrays coupled with a dense array of detectors (and sources) for light harvesting (and injection) or the integration of optic and electronic components in a hybrid chip in order to produce an optical feedback loop directly on the chip for monitoring the instrument status.

\subsection{Detectors}

The unceasing trend toward reliable, low-cost, and compact TD diffuse optics systems is fostering the interest in solid-state single-photon detectors as the most valuable alternative to traditional sensors. Photocathode-based devices like PMTs, MCPPMTs, or hybrid PMTs ${ }^{99}$ are indeed intrinsically bulky and not scalable due to their structure based on the separation in a vacuum tube between the cathode and the electron multiplication region. Additionally, the presence of electromagnetic fields can prevent their use in particular environments [e.g., close to magnetic resonance imaging (MRI) systems] and the exposure to mechanical shocks or normal room light can easily be lethal for the device. Finally, they require a biasing voltage of few $\mathrm{kV}$ to trigger the electron multiplication process and they are quite expensive due to the fabrication process.

On the other hand, solid-state detectors like SPADs are not affected by previous disadvantages, but up to now, they have rarely been the first choice for TD diffuse optics systems due to their small active area diameter of few hundreds of micrometers in the best case. ${ }^{100,101}$ Devices with an active area diameter up to $0.5 \mathrm{~mm}$ recently have been demonstrated, but their timing performance is still unsatisfactory for this application. ${ }^{102}$

Diffuse optics intrinsically requires detectors with a large active area to maximize light harvesting since, differently from collimated light, diffused photons cannot be focused without significant losses into a small area. Similarly, when largecore fibers or fiber bundles are employed to collect diffused photons, a small area device still represents a bottleneck for the light harvesting efficiency. Therefore, photon detection efficiency (PDE) alone is insufficient to provide information on the photon collection effectiveness of a detection chain. Within the recent activity on standardization of the characterization protocols for photon migration instruments, ${ }^{63,103,104}$ the so-called "responsivity" (i.e., overall PDE from a Lambertian source) has been defined in order to have a figure of merit providing direct information about the overall efficiency in detecting light emerging from tissues. Responsivity indeed depends not only on PDE but also on the NA of the optical system and on the effective collection area. Due to the small active area of commercially available SPADs, the responsivity of a detection chain based on this technology is typically tens or hundreds of times lower than in a PMT-based system, ${ }^{105}$ thus reducing the SNR of the measurement.

At any rate, this technology has been considered within recent years ${ }^{69-72,97,106,107}$ because it is the only one presently allowing the exploration of new physical concepts like the null source-detector separation and measurement schemes based on the dense arrangement of optodes. ${ }^{73}$ We already discussed in Sec. 2 the requirement of a time-gated acquisition system in order to allow a small or null separation geometry by rejecting early photons. Even if time-gating of photocathodebased detectors is feasible by applying a fast voltage transition to the signal amplification stage to suppress the electron multiplication process, ${ }^{108-112}$ or again by properly acting on the ramp voltage of a streak camera in order to sweep undesired photoelectrons out of the phosphor screen, ${ }^{113}$ the photocathode is always hit by the burst of unwanted early light, thus possibly damaging it or at least demoting its performance in terms of background noise. This problem is not easily noticed using a SPAD, where usually millions of carriers can flow within the junction without any problem for the detector. ${ }^{114}$ In a SPAD, a single-photon impinging on the active area can generate a single electron-hole pair, which is accelerated by the high electric field (well above the breakdown level), thus possibly triggering an avalanche current pulse in the $\mathrm{mA}$ range since the current is limited by the front-end circuit at a level well below the damaging threshold. ${ }^{115}$ Therefore, usually, the light exposure cannot have a permanent detrimental effect on the detector.

When operated in the time-gated mode for TD diffuse optics, the SPAD biasing voltage is kept below breakdown in correspondence of early photons; then, it is swiftly increased above breakdown (e.g., transition time $<200 \mathrm{ps}$ ) to be able to detect late photons. ${ }^{116-118}$ In this way, the early light exiting from the tissue will generate millions of electron-hole pairs without affecting the detector performance at late times. However, in the case of very strong light exposure during the gate-off time (e.g., $>1 \mathrm{~mW}$ ), the background noise starts to increase, thus setting a limit of about 8 orders of magnitude to the maximum achievable dynamic range in time-gated measurements. ${ }^{119}$ Such a value is much higher than the typical dynamic range that can be obtained by employing a detection chain based on standard free-running (i.e., not-gated) detectors and short integration times. Simulations demonstrated that an unreasonable integration time of more than 3 years would be required for a free-running SPAD to obtain the same dynamic range that be achieved in just $1 \mathrm{~s}$ using a fast-gated one. ${ }^{120}$ The realization of fast time-gated SPAD detectors has been possible due to the evolution of the front-end electronic circuits. ${ }^{121}$ Different solutions have been proposed in the literature for time-gated microelectronic detectors for telecommunication wavelengths, ${ }^{122-128}$ but the fast transitions required by diffuse optics at null-distance, together with the requirement for a flat detection efficiency of the detector just after the fast transition, required a dedicated development. ${ }^{116}$

Besides responsivity and the ability to operate in time-gating, the detector response tail is another feature particularly important for properly probing highly scattering media at small source-detector separations. In a SPAD, photons can be absorbed either within the depleted high-electric field region (thus, promptly triggering the avalanche of carriers) or within the neutral region close to the active junction. ${ }^{129}$ In this latter case, the avalanche triggering is delayed since photogenerated carriers have to move at first by diffusion before reaching the high field region, thus giving rise to an exponential decay tail in the IRF (typically from tens up to hundreds of picosecond time constant) that can lower the performances in both the recovery of the optical properties and in the investigation of heterogeneous media. ${ }^{130}$ Presently, the problem has been solved due to an accurate selection of the production run ( $~ 90$-ps decay time constant), ${ }^{116}$ but future research must cope with this feature in tailoring novel devices for the specific application.

As mentioned earlier, SPADs also enabled the concept of a dense arrangement of measurement points to maximize the SNR. ${ }^{73}$ Similar to the situation already described for VCSELs, a SPAD requires in principle a quite simple front-end electronics, which can be integrated into a single chip that can be ideally placed close to the SPAD die or behind it using 3-D integrated 
circuit arrangements. In this way, many SPADs can be arranged into a small area with high density. Furthermore, they can be placed directly in front of the tissue under investigation, thus avoiding the use of either cumbersome fiber bundles or additional optics elements that could introduce both additional losses and spread in time. In this latter case, the gain in responsivity is very high (around 1 order of magnitude) due to the high NA of the detector (close to 1) that improves diffused light harvesting with respect to the use of any optics or optical fibers. ${ }^{73}$

It is worth now to point out that SPADs can be realized using different technologies. Generally speaking, it is possible to distinguish between custom-technology SPADs and complementary metal-oxide-semiconductor (CMOS) SPADs. ${ }^{131}$ In the former case, performances are usually better (e.g., PDE and noise level), but the required driving electronics cannot be directly integrated on the same SPAD die. The latter technology usually features slightly lower performances, but it allows the integration of the whole electronic circuitry on the same die of the SPAD. Therefore, CMOS SPAD technology easily allows the realization of large arrays of thousands of cells, each one containing a single SPAD and its own basic front-end circuitry and sometimes also counters or TDCs for the generation of the photon time stamps. ${ }^{132}$ Unfortunately, the use of large SPAD arrays in TD diffuse optics to cope with the low responsivity of the single SPAD is not truly effective since the ratio between the active area of the detector and the area of the silicon die used for the additional electronics is typically small (a few percent), thus losing the majority of photons emerging from the tissue.

It is evident at this point that the ideal single-photon detector for TD diffuse optics is a solid-state detector, but with the wide active area typical of photocathode-based devices. At present, the only available solution is represented by silicon photomultipliers (SiPM) also known as multipixel photon counters and in many other ways depending on the producer. ${ }^{133,134}$ Essentially, its basic structure is similar to an array of hundreds or thousands of SPADs, all connected in parallel with a high fill factor (e.g., $>60 \%$ ), giving a single-global anode and a single-global cathode contact. Additionally, each SPAD is equipped with an integrated quenching resistor of different hundreds $\mathrm{k} \Omega$, which passively quenches the avalanche ignition triggered by a photon and then passively restores the previous biasing condition. ${ }^{100}$ The passive quenching/restoring scheme is widely recognized to be slow and ineffective, thus limiting the maximum count rate and increasing the background noise of the detector due to afterpulsing. ${ }^{135}$ However, those effects are mainly due to the parasitic capacitance of the resistor terminal, ${ }^{121}$ which is almost negligible when the resistor is integrated. Additionally, every time a cell is fired by a single photon, the device outputs a voltage pulse, while other thousands of cells remain ready to detect other photons, thus allowing very high count rates up to a billion counts per second (cps). ${ }^{136,137}$ SiPMs were originally developed as photon-number resolving detectors exploiting their parallel structure that allows outputting a voltage pulse almost proportional to the number of photons simultaneously impinging onto the detector. Indeed, their development has been strongly pushed ahead by high-energy physics applications and positron emission tomography (PET) scanners, where they are used to detect light triggered by charged particles or ionizing radiation into scintillators. ${ }^{138}$ However, their use in single-photon counting and timing applications has long been assumed unfeasible in particular due to the high-background noise of some Mcps for a $1-\mathrm{mm}^{2}$ device and discordant data about timing resolution (from few hundreds of ps to $>1 \mathrm{~ns}) .{ }^{139,140}$ However, due to the highly demanding applications with different links to diffuse optics requirements (e.g., large area, low noise, high timing resolution, and buttable geometry to parallelize many measurement points), the SiPM technology recently evolved toward improved performance and very low cost (e.g., few tens $€$ for a single device). ${ }^{141-143}$ Indeed, during the last 3 years, SiPM started to be considered for CW diffuse optics prototypes ${ }^{144,145}$ and last year, their use was also demonstrated in the TD regime, ${ }^{146}$ mainly due to a targeted selection of high-performance devices and to the development of a compact and suitable electronic solution to maximize the timing resolution. ${ }^{147} \mathrm{SiPMs}$ have demonstrated more than 1 order of magnitude improvement in responsivity with respect to state-of-the-art devices, ${ }^{146}$ with high temporal resolution $\left(\sim 60\right.$ or $\sim 130$ ps for 1.7 - and $9-\mathrm{mm}^{2}$ devices, respectively, presently, the fastest response reported in the literature) and acceptable dark count rate $\left(\sim 10^{5} \mathrm{cps} / \mathrm{mm}^{2}\right)$, thus opening the way to novel outlooks in the field with fascinating perspectives toward wearable and low-cost devices. Their clinical exploitation is essentially starting right now due to the realization of the first clinical broadband diffuse optical spectrometer, which already demonstrated its potential in clinical setting in the measurement of different bony prominences of the human body as a pilot step toward noninvasive assessment of bone pathologies, ${ }^{105,148}$ and of the first prototype of clinical system for in-vivo brain oximetry. ${ }^{149}$

Unfortunately, the present technology of SiPM does not allow the fast-gated operation, ${ }^{150}$ thus preventing small sourcedetector separation measurements. This is another point that the future research has to address, maybe due to the use of the CMOS process to realize novel SiPM architectures, ${ }^{151}$ since in this case, it is also possible to integrate custom electronics in each pixel at the expense of the fill factor.

Solid-state technology has also permitted to explore in diffuse optics the spectral region beyond $1100 \mathrm{~nm}$, where strong differences are expected among different tissues components, but little data are available in scattering media. The extension of clinical systems beyond $1100 \mathrm{~nm}$ is expected to provide an important contribution for the quantification of, for example, collagen (for breast cancer risk assessment), glucose (for monitoring diabetes), or hydroxyapatite (for osteoporosis diagnosis). Unfortunately, time-resolved detectors for the NIR region like PMTs are affected by additional disadvantages like low quantum efficiency (e.g., $\sim 0.5 \%$ ) and high transit time spread (>1 ns), while, due to the high absorption of water at wavelengths longer than $1000 \mathrm{~nm}$, both quantum efficiency and timing resolution become particularly important: the former because of the high attenuation that affects the total amount of photons exiting from the tissue; the latter because a high absorption coefficient produces narrow waveforms of remitted photons, thus approaching the width of the IRF and possibly preventing the possibility to properly recover optical properties. ${ }^{103}$ A possible alternative is offered by MCP-PMTs for the NIR region, which still present low quantum efficiency but much narrower time jitter $(<100 \mathrm{ps})$, and they have been successfully used for TD diffuse optics systems up to $1400 \mathrm{~nm} .{ }^{152}$

Also for the NIR region, SPADs are truly attractive due to their high quantum efficiency (30\% to $40 \%$, i.e., about 2 orders of magnitude higher than photocathode-based devices) and low time jitter ( $<50 \mathrm{ps})$, but again, their small active area (typically $<50 \mu$ m diameter, i.e., 3 or 4 orders of magnitude smaller than those of a NIR-PMT) limits the photon collection efficiency. ${ }^{153,154}$ 
In this case, the device is much more complex with respect to silicon SPADs since the absorption region is typically made of InGaAs to absorb NIR radiation, while the avalanche process takes place in another lattice-matched layer with a larger energy band gap (e.g., InP or InAlAs) in order to avoid to have high electric fields within a low-energy band-gap region since this combination would generate a very high dark count rate due to field-assisted noise generation processes. These detectors also require moderate cooling with, e.g., a Peltier stage, to limit the spontaneous thermal generation. Noticeably, a compact TD diffuse optics system working up to $1700 \mathrm{~nm}$ based on an InGaAs/InP SPAD has been built up, with successful proof-ofconcept measurements on lipid, ${ }^{155}$ collagen, ${ }^{156}$ and in vivo. ${ }^{157}$

In order to provide solid-state single-photon detectors with a high photon counting rate and photon-number resolving capability also for the NIR range, an architecture similar to SiPM has been proposed also for InGaAs SPADs, under the name of negative feedback avalanche diode. ${ }^{158,159}$ Such a device has been produced in arrays of tens of detectors, ${ }^{160}$ but the fill factor is still quite low, being similar to arrays of SPADs. However, this technology seems to be promising in terms of performances to allow in the future to replace photocathode-based detectors also in the NIR region, thus prefiguring broadband diffuse optical spectrometers with extended spectral region with the combination of silicon and III-V detectors technologies.

\subsection{Timing Electronics}

In a TD system, the detector provides a voltage pulse that marks the arrival time of a detected photon. After that, a final stage provides a digital number that encodes such time with respect to the synchronization signal. This time-stamp can be used to build up a histogram of the arrival times of detected photons, thus recovering the time-resolved curve shape for further analysis. The technique is named TCSPC. ${ }^{99,161,162}$ Similar to previous components, also in this case, the main impairments are high cost, high power dissipation, and limited performance (e.g., conversion time, linearity, time resolution, and so on).

Generally speaking, there are two main strategies for designing a timing stage: one is based on a time-to-analog converter (TAC) and the other one is based on a TDC. ${ }^{99,163}$ In the former case, the time difference between the start and the stop pulse is converted into a voltage amplitude, which is then processed by an analog-to-digital converter (ADC) to obtain the digital code related to the delay. In the latter case, the whole process is digital and based on the combination of suitable delay lines and digital counters. Usually, analog technologies perform better in terms of timing resolution, but are lacking in integration capability, thus forcing the use of discrete electronic components. On the contrary, a TDC can be integrated into a single chip, thus easily allowing multichannel devices, but the timing performances in terms of both resolution and linearity are usually worse.

The first generation of TCSPC electronics, used since the early 1970 s for the measurement of fluorescence decays, was based on bulky rack-hosted NIM devices. The complete timing electronics was essentially composed of the combination of three modules: TAC, ADC, and a multichannel analyzer to generate the histogram of the arrival times. With these solutions, the maximum conversion rate (expressed as counts per second, cps) was limited between few tens of kcps to about $100 \mathrm{kcps} .{ }^{161}$

A modern solution, commonly used in TD diffuse optics systems, is the use of either a PC board based on TACs and ADCs ${ }^{99,164}$ or a bench module based on TDCs. ${ }^{165}$ Notwithstanding the differences between these two technologies, they show similar performances in terms of minimum timing jitter $(\sim 10 \mathrm{ps})$, nonlinearity $(\sim 1 \%)$, maximum count rate (few Mcps), power consumption (tens of watts), and cost (few k€/channel). However, the recent developments in TDC chips provide promising new solutions for the next generation of diffuse optics systems with the potentiality to reduce costs and provide compact and low power technologies able to be integrated into future wearable devices. ${ }^{166,167}$ Indeed, arrays of thousands of SPADs with in-pixel TDC ${ }^{132,168-170}$ or alternatively with a battery of TDCs shared among different pixels ${ }^{171,172}$ are being developed in cost-effective CMOS process. These solutions have just started to be applied on phantoms in TD diffuse optics systems and are providing promising results. ${ }^{173}$

A very interesting alternative is represented by the so-called "digiSiPM," which is similar to a standard SiPM, but integrates one (or sometimes more) TDC. ${ }^{174}$ In this case, the SiPM-like architecture potentially guarantees high responsivity, while the integrated TDC allows to minimize cost, size, and power consumption. However, the dark count rate of this kind of detector is still too high to be fully suitable for real application, being of the order of a few Mcps $/ \mathrm{mm}^{2}$. Considering that, in order to limit the pile-up distortion when using a TCSPC approach, there is the need to keep the conversion rate of the detection chain in the order of few percent of the laser repetition rate (typically, $<100 \mathrm{MHz}),{ }^{175}$ the dark count rate alone may completely saturate the maximum count rate of the TDC. While waiting for an improvement of the technology in terms of noise, other possible solutions are emerging like novel digiSiPM architectures with banks of tens of TDCs shared among different cells of the SiPM, thus potentially improving the maximum counting rate. ${ }^{176,177}$ Indeed, in this case, the detection chain is essentially split into parallel chains, thus breaking the limit of the pile-up distortion: every time a single cell of the SiPM is fired, one of the TDCs starts to compute the time-stamp, but in the meanwhile, thousands of other cells are ready to detect other photons and tens of other TDCs are ready to start other conversions. Therefore, multiple photons can be properly processed for each laser pulse instead of just one like in more traditional solutions, thus reaching conversion rates up to $\sim 1$ Gcps on a single detection stage, with a potential breakthrough in the SNR of the measurement. ${ }^{178,179}$ However, the applicability of novel digiSiPMs in TD diffuse optics systems is still to be demonstrated, since, up to now, they have been mainly applied in fluorescence decay measurements.

Recently, a possible alternative approach has been proposed to increase the count rate, based on a dual-window fast-gated counter to acquire the remitted photons at both early and late delays, but in well-defined time-windows. ${ }^{180}$ This approach is useful in applications where the full waveform shape is not needed, as, for example, in brain or muscle imaging applications, where the early window monitors systemic activity at the level of the skin and the late window extracts information about deeper structures. ${ }^{64-67}$ In the present implementation, the fast-gated counter allows acquiring up to $100 \mathrm{Mcps}$ (ten times higher than the limit of the traditional TCSPC systems), but holding the potential of reaching $\mathrm{GHz}$ counts rate with proper electronic design, without loss of performance with respect to a full TCSPC system. ${ }^{181}$ Additionally, a fast-gated counter can also be easily integrated in a single die, thus allowing low cost, low power, and highly scalable and parallelizable solutions for next-gen TD systems. 


\section{Future Applications and Vision}

In the previous sections, we presented methods and technologies that have been used in laboratory settings for proof-of-principle studies on phantoms or for pilot in vivo studies. Therefore, unlike the previous generations, the next-gen TD systems still have not been thoroughly tested at the clinical level. A singlechannel clinical tissue oximeter using TD NIRS based on SiPM and TDC has been recently developed and approved as a medical device in Japan (tNIRS-1, Hamamatsu Ltd.). ${ }^{149}$ We expect that other clinical TD systems will be released from research groups in the coming years. The full exploitation of the potentialities offered by the physics of TD diffuse optics and by the new photonic components paves the way to new or more effective applications and creates a fascinating long-term vision on the possible evolution of the whole field.

A first direction is toward deep sensing. As depicted in Fig. 4(a), under realistic (and safe) limits of laser exposure and light harvesting, the maximum penetration depth can be considerably increased (by at least a factor of 2), as compared to the actual limits, making it possible to assess structures deeper than 5 to $6 \mathrm{~cm} .{ }^{73} \mathrm{New}$ organs and functions could be accessible. One of the most attractive is the heart, with the possibility to monitor continuously its perfusion status and foresee the early onset of infarct. Ischemic zones in the heart can be easily mapped by TD NIRS during chest surgery, ${ }^{182}$ and the quest would be to get the same information noninvasively transthoracic. Also, the lung - at the center of many pathologies and morbidities-is a hidden organ, which could be within the reach of TD NIRS. In a preliminary experiment, respiration-related changes in the optical signal possibly ascribed to the changes at the lung level were observed using a laboratory system. ${ }^{183}$ Furthermore, fetal brain monitoring, which was proposed in clinical case studies ${ }^{184}$ or in animal models, ${ }^{185}$ could gain wider impact by increasing the depth of sight and quantitation accuracy of new systems. Obviously, the broad field of brain imaging and neuromonitoring will take advantage of increased penetration depth, for accessing subcortical structures as for traumatic brain injury or stroke, as well as of increased sensitivity at the cortex level by extraction of late photons. In general, looking back at Fig. 4(a), for a typical depth of 2 to $3 \mathrm{~cm}$, the full exploitation of TD physics could lead the transition from a sensitivity level just above the threshold of visibility up to more robust and reliable signals.

Multimodality approaches in diffuse optics are gaining interest because of the possibility to combine morphological evidence with the specific chemical and functional information provided by optics, and because of the great improvement in quantitation, whenever the morphological map is used as a priori constrain for optical reconstruction. Several approaches have been already proposed by combining diffuse optics with x-rays, MRI, electroencephalography (EEG), and ultrasound. ${ }^{1,186}$ In this latter case, the possibility to operate at null or short sourcedetector distance by TD approaches is a great plus toward the deployment of handheld hybrid probe compatible with standard ultrasound use. Indeed, TD optical tomography operates in a similar way as ultrasound, since the echoes of the photon density waves scattered by tissue heterogeneities are detected back at the surface as perturbations over the diffusely reflected signal. By increasing the photon delay time, echoes from deeper regions are observed.

In a different direction, but still with the goal to increase the sensitivity and specificity of optical tools, we can observe the extension of diffuse optics toward expanded spectral ranges both at lower $(<600 \mathrm{~nm})$ and higher $(>1000 \mathrm{~nm})$ wavelengths. ${ }^{155,187}$ The quest here is to be able to track specific markers of pathology or functions. A first example is collagen, whose fingerprint is mainly in the 1000- to $1100-\mathrm{nm}$ region, which could help in discriminating breast cancer lesions ${ }^{188}$ and identify a risk factor for breast cancer onset. ${ }^{189-191}$ Extending the spectral range up to $1700 \mathrm{~nm}$, or down to $500 \mathrm{~nm}$, will permit to detect absorbers with a negligible contribution in the classical therapeutically window. Surely, this spectral region has already been largely explored by classical NIRS, with many applications, particularly in the industrial sector. ${ }^{37}$ Still, TD NIRS will bring absolute quantitation and increased depth penetration, which are two limiting factors in typical NIRS approaches. The spectral expansion of TD NIRS is now possible because of the concomitant achievement of new broadly tunable laser sources (see Sec. 3.1), InGaAs detectors (see Sec. 3.3), and advanced solutions of the radiative transport equation overcoming the limits of the diffusion approximation for high absorption. ${ }^{192,193}$

The three previous paragraphs address the increase in information content achievable with next-gen TD NIRS. Alongside with this, also the expansion of usability and proposal of new modalities are also expected. A first example is the introduction of wearable devices. These tools have been already developed for the CW domain. ${ }^{76}$ Still, wearable TD devices could provide more robust signals because of the higher tolerance to moving artifacts and superficial effects, the larger independence from background signal, and the possibility to get information out of a single optode by hosting the source and detector at null or small relative distance. ${ }^{194}$ As discussed, new chip-based photonic components, such as the pulsed VCSEL as source, SIPM for detector, and CMOS TDC, are perfect ingredients for smart wearable devices to be used in brain monitors or muscle oximeters.

A further perspective is offered by the noncontact approach. Proof-of-concept phantom measurements and initial in vivo tests during motor and cognitive brain activation paradigms have shown the feasibility of remote noncontact deep tissue imaging. ${ }^{71,97}$ The clue here is the adoption of fast gating to extract deep signals out of the overwhelming contamination of direct reflections and superficial (extracerebral) signals. Coupled with scanning ${ }^{71}$ or smart tracking systems, this approach is attractive for intraoperative subsurface imaging or remote sensing. Generally, it can make the detection of cognitive functions more appealing since no probe positioning or dedicated helmets are needed. Should the hemodynamic response of the brain be proven as a valid indicator of lie detection as suggested in the past, ${ }^{195-199}$ then noncontact diffuse optics systems could be employed also for security installations.

Key issues are surely miniaturization and tremendous cost reduction. Next-gen TD systems could be easily produced as smart all-integrated microelectronic devices using standard technologies or exploiting low-cost production of standard telecom components (e.g., VCSELs). Since the electronics integrating the photonic components can be designed as an ASIC, the production of a smart TD NIRS chip is industrially feasible. The driving force here could be the consumer market since TD NIRS is possibly the only technology compatible with safe and easy consumer use, capable to provide functional information from inside the human body. As pointed out by key technology players, ${ }^{92}$ it is likely that future smartphones will host onboard many VCSELs for increased interactions with the environment and the people. Using a smartphone for remote 
noncontact sensing of the subject's head for new dimensions of augmented reality is not out of sight. Also, the longed-for remote home diagnostics could achieve a new tool at hand by quick extraction of functional parameters to be sent by the patient to a remote medical expert.

Cost and size reduction, wearable probes, and intrinsic robustness of TD NIRS from external ambient contamination (e.g., moving artifacts, background light, and displacements) reveal new perspectives also for sociopsychological studies, like hyperscanning. ${ }^{200}$ Simultaneous monitoring of brain responses in different subjects performing the same protocols or interacting with each other has been approached with functional magnetic resonance imaging (fMRI) and with EEG. However, due to the constraints associated to fMRI, the combined approach fMRI-EEG is restricted to protocols not involving freedom of movement to the subject, therefore limiting the wealth of studies. The idea to distribute many TD NIRS head probes to individuals interacting in common tasks (e.g., students listening to a lecture or people watching a football match) is fascinating for studying the reaction of different brains to the same stimuli and for understanding the roles of interaction and environment. Photonics could help in bridging the world of social sciences/anthropology/sociology and psychology/pedagogy to the world of neurology/neurosciences by providing simple tools to quantify brain functions in real-life operations. Clearly, behind these dreams, which are in principle within the capabilities also of $\mathrm{CW}$ devices, there is the compelling need to improve the accuracy and reliability of NIRS signals, to get rid of all confounding effects. Again, referring to Sec. 2, this goal can be reached fully exploiting the TD approach by a combination of a 10-fold increase in sensitivity to cortex activations using late photons and of the disentanglement from superficial/systemic and environmental effects.

Another potential field is offered by ethology. Assessing brain functions noninvasively in animals (e.g., sheep, dog) opens new perspectives in a field, where fMRI is severely hampered and limited to few well-trained animals. Some promising results have been presented using wearable $\mathrm{CW}$ devices, ${ }^{201-204}$ and the new TD technology could yield further advances. This is a unique path to better understand their evolution and behavior but also to monitor distress or suffering in intensive breeding or in animal experiments. Due to increasing public opinion awareness, ${ }^{205}$ this aspect could become critical in the future also on the normative side.

The prefigured advancements of TD diffuse optics — some of them already at a proof-of-principle stage_-are extremely timely since they meet the user and clinical needs for new smart tools to probe the body functions in depth, which are raising great interest from strong technology actors. ${ }^{206}$ As shown in Table 1, nextgen TD NIRS will offer within the same technology a wealth of key features - some of them already well established, others new or enhanced-which could be definitely of interest in the search of novel personalized sensing tools.

"Noninvasiveness" is the most obvious feature and at the basis of the key attractiveness of photonics. This, of course, is a key requirement to serve also the market of remote home diagnostics. "Noncontact" is made possible by the adoption of shortdistance time-gated measurements and expands the field of applicability of TD NIRS to new scenarios. "Functional and chemical capabilities" are a well-known benefit of optics as compared to most of the traditional clinical imaging modalities, which rely mostly on morphology. "Wearability" is a consequence of the continuous process of miniaturization. Although not yet proven for TD, the key components are already there and could be achieved with proper engineering. "Depth sensitivity" is definitely a crucial advantage of diffuse optics over all other optical diagnostics tools. As shown in Fig. 4(a), we are still far from the ultimate limits, and the foreseen increase in sensitivity will secure existing applications on sounder basis. "Absolute quantitation" of the absorption coefficient-and of the related tissue composition - is a good requisite for an objective grading of a lesion or of a response. Finally, "scalability" comes from the

Table 1 Main feature of TD diffuse optics.

Description Status References

Noninvasive Completely noninvasive and nondestructive (for industrial applications)

Noncontact Remote noncontact monitoring feasible either as singlepoint or in scanning mode

Functional Provide both functional (i.e., related to the metabolism or oxygen demand) and chemical (i.e., related to tissue composition) information

Wearable Wearable devices feasible for point-of-care, personalized diagnostics or continuous monitoring

Deep Probing subsurface tissues down to a depth of around $6 \mathrm{~cm}$

Operator Absolute automatic quantification not dependent on independent skilled personnel

Scalable The very same basic unit is used on single-optode smartphone-based consumer applications and on $>1000$ optodes PET-like clinical systems
Limits of TD NIRS are calculated for the maximum safe laser exposure

Proof-of-concept on phantoms and on basic brain activation paradigm

This is a well-known advantage of optics as compared to most of other purely morphological imaging modalities in clinics

Already demonstrated for CW, not yet for TD

Deep probing up to $9 \mathrm{~cm}$ source-detector separation with TD NIRS, only simulations for short distances

73, 207

Some attempts toward, e.g., classification of breast lesions

Usability of basic building blocks embedded onto the probe demonstrated

73

71,97

1

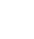


sharing of the same physical basis and elementary TD cell between a $>1000$ optode PET-like clinical optical tomograph and a single-optode smartphone-based device. Taken as a whole, all these distinct features, not easily found even separately in other diagnostics modalities, prefigure a new pervasive and unique technology, which could result in one of the most attractive photonic tools of next decades.

\section{Conclusion}

We have reviewed the basic features of the TD approach to diffuse optics and the building blocks of TD systems. The recent advances in photonic components and devices enable the exploitation of the new physical concepts of time-gating and null distance. The vision for the next 20 years is that TD NIRS and DOI systems will become smaller, at the level of integration in wearable devices, and smarter, increasing their overall accuracy in the noninvasive detection and quantification of tissue components. Clinical-, industrial-, and consumer-level applications will benefit from this advanced technology.

\section{Acknowledgments}

The research leading to these results has partially received funding from the European Commission under the projects BabyLux (Grant Agreement Nos. 620996, CIP-ICT-PSP2013-7), LaserLab Europe (Grant Agreement Nos. 654148, H2020-INFRAIA-2014-2015), and LUCA (Grant Agreement Nos. 688303, H2020-ICT-2015).

\section{References}

1. T. Durduran et al., "Diffuse optics for tissue monitoring and tomography," Rep. Prog. Phys. 73(7), 076701 (2010).

2. A. P. Gibson and H. Dehghani, "Diffuse optical imaging," Philos. Trans. A: Math. Phys. Eng. Sci. 367(1900), 3055-3072 (2009).

3. D. T. Delpy and M. Cope, "Quantification in tissue near-infrared spectroscopy," Philos. Trans. R. Soc. London. Ser. B, Biol. Sci. 352(1354), 649-659 (1997).

4. H. Liu et al., "Determination of optical properties and blood oxygenation in tissue using continuous NIR light," Phys. Med. Biol. 40(11), 1983-1993 (1995).

5. A. Kienle, F. K. Forster, and R. Hibst, "Influence of the phase function on determination of the optical properties of biological tissue by spatially resolved reflectance," Opt. Lett. 26(20), 1571-1573 (2001).

6. T. Durduran et al., "Bulk optical properties of healthy female breast tissue," Phys. Med. Biol. 47(16), 2847-2861 (2002).

7. P. Taroni et al., "In vivo absorption and scattering spectroscopy of biological tissues," Photochem. Photobiol. Sci. 2(2), 124-129 (2003).

8. A. Pifferi et al., "Spectroscopic time-resolved diffuse reflectance and transmittance measurements of the female breast at different interfiber distances," J. Biomed. Opt. 9(6), 1143-1151 (2004).

9. V. Chernomordik et al., "Quantification of optical properties of a breast tumor using random walk theory," J. Biomed. Opt. 7(1), 80-87 (2002).

10. A. Liebert et al., "Evaluation of optical properties of highly scattering media by moments of distributions of times of flight of photons," Appl. Opt. 42(28), 5785-5792 (2003).

11. A. Yodh and B. Chance, "Spectroscopy and imaging with diffusing light," Phys. Today 48(3), 34-40 (1995).

12. A. P. Gibson, J. C. Hebden, and S. R. Arridge, "Recent advances in diffuse optical imaging," Phys. Med. Biol. 50(4), R1-R43 (2005).

13. D. T. Delpy et al., "Estimation of optical pathlength through tissue from direct time of flight measurement," Phys. Med. Biol. 33(12), 1433-1442 (1988).

14. M. S. Patterson, B. Chance, and B. C. Wilson, "Time resolved reflectance and transmittance for the non-invasive measurement of tissue optical properties," Appl. Opt. 28(12), 2331-2336 (1989).

15. S. L. Jacques, "Time resolved propagation of ultrashort laser pulses within turbid tissues," Appl. Opt. 28(12), 2223-2229 (1989).
16. B. Chance et al., "Comparison of time-resolved and -unresolved measurements of deoxyhemoglobin in brain," Proc. Natl. Acad. Sci. U. S. A. 85(14), 4971-4975 (1988), http://www.pnas.org/content/85/14/4971 .abstract.

17. M. Ferrari et al., "Time-resolved spectroscopy of human forearm," J. Photochem. Photobiol. B 16(2), 141-153 (1992).

18. R. Cubeddu et al., "Time-resolved reflectance: a systematic study for application to the optical characterization of tissues," IEEE $J$. Quantum Electron. 30(10), 2421-2430 (1994).

19. S. Andersson-Engels et al., "Multispectral tissue characterization with time-resolved detection of diffusely scattered white light," Opt. Lett. 18(20), 1697-1699 (1993).

20. J. C. Hebden, "Evaluating the spatial resolution performance of a timeresolved optical imaging system," Med. Phys. 19(4), 1081-1087 (1992).

21. I. Oda et al., "Optical tomography by the temporally extrapolated absorbance method," Appl. Opt. 35(1), 169-75 (1996).

22. R. Cubeddu et al., "Imaging of optical inhomogeneities in highly diffusive media: discrimination between scattering and absorption contributions," Appl. Phys. Lett. 69(27), 4162-4164 (1996).

23. C. Lindquist et al., "Reconstruction of diffuse photon-density wave interference in turbid media from time-resolved transmittance measurements," Appl. Phys. Lett. 69(12), 1674-1676 (1996).

24. W. Cui, N. Wang, and B. Chance, "Study of photon migration depths with time-resolved spectroscopy," Opt. Lett. 16(21), 1632-1634 (1991).

25. A. Torricelli et al., "In vivo optical characterization of human tissues from 610 to $1010 \mathrm{~nm}$ by time-resolved reflectance spectroscopy," Phys. Med. Biol. 46(8), 2227-2237 (2001).

26. H. Heusmann, J. G. Koelzer, and G. Mitic, "Characterization of female breasts in vivo by time-resolved and spectroscopic measurements in the near infrared spectroscopy," J. Biomed. Opt. 1(4), 425-434 (1996).

27. R. Cubeddu et al., "Noninvasive absorption and scattering spectroscopy of bulk diffusive media: an application to the optical characterization of human breast,” Appl. Phys. Lett. 74(6), 874-876 (1999).

28. R. Cubeddu et al., "Compact tissue oximeter based on dual-wavelength multichannel time-resolved reflectance," Appl. Opt. 38(16), 3670-3680 (1999).

29. D. Grosenick et al., "Development of a time-domain optical mammograph and first in vivo applications," Appl. Opt. 38(13), 2927-2943 (1999).

30. V. Ntziachristos, X. Ma, and B. Chance, "Time-correlated single photon counting imager for simultaneous magnetic resonance and near-infrared mammography," Rev. Sci. Instrum. 69(12), 4221-4233 (1998).

31. H. Eda et al., "Multichannel time-resolved optical tomographic imaging system," Rev. Sci. Instrum. 70(9), 3595-3602 (1999).

32. F. E. W. Schmidt et al., "A 32-channel time-resolved instrument for medical optical tomography," Rev. Sci. Instrum. 71(1), 256-265 (2000).

33. T. Yates et al., "Optical tomography of the breast using a multi-channel time-resolved imager," Phys. Med. Biol. 50(11), 2503-2517 (2005).

34. A. Torricelli et al., "Recent advances in time-resolved NIR spectroscopy for nondestructive assessment of fruit quality," Chem. Eng. Trans. 44(1), 43-48 (2015).

35. D. Grosenick et al., "Optical breast imaging and spectroscopy," J. Biomed. Opt. 21(9) (2016).

36. A. Torricelli et al., "Time domain functional NIRS imaging for human brain mapping," Neuroimage 85(1), 28-50 (2014).

37. D. Contini et al., "Review: brain and muscle near infrared spectroscopy/ imaging techniques," J. Near Infrared Spectrosc. 20(1), 15-27 (2012).

38. R. L. van Veen et al., "Determination of visible near-IR absorption coefficients of mammalian fat using time- and spatially resolved diffuse reflectance and transmission spectroscopy," J. Biomed. Opt. 10(5), 054004 (2005).

39. A. Pifferi et al., "Optical biopsy of bone tissue: a step toward the diagnosis of bone pathologies," J. Biomed. Opt. 9(3), 474-480 (2004).

40. P. Taroni et al., "Absorption of collagen: effects on the estimate of breast composition and related diagnostic implications," J. Biomed. Opt. 12(1), 014021 (2007).

41. A. Torricelli et al., "Time-resolved reflectance spectroscopy for nondestructive assessment of food quality," Sens. Instrum. Food Qual. 2(2), 82-89 (2008).

42. I. Bargigia et al., "Diffuse optical techniques applied to wood characterization," J. Near Infrared Spectrosc. 21(4), 259-268 (2013). 
43. C. Abrahamsson et al., "Time-resolved NIR spectroscopy for quantitative analysis of intact pharmaceutical tablets," Anal. Chem. 77(4), 10551059 (2005).

44. F. Kamran et al., "Transmission near-infrared (NIR) and photon time-offlight (PTOF) spectroscopy in a comparative analysis of pharmaceuticals," Appl. Spectrosc. 69(3), 389-397 (2015).

45. D. Khoptyar et al., "Broadband photon time-of-flight spectroscopy of pharmaceuticals and highly scattering plastics in the VIS and close NIR spectral ranges," Opt. Express 21(18), 20941-20953 (2013).

46. C. D'Andrea et al., "Absorption spectroscopy of powdered materials using time-resolved diffuse optical methods," Appl. Opt. 51(32), 7858-7863 (2012).

47. Hamamatsu Photonics, K.K., "Biomedical measurement using light," http://www.hamamatsu.com/us/en/hamamatsu/overview/bsd/central_ research_laboratory/health_and_medical_care_field.html (06 June 2016).

48. AUREA Technology SAS, "PICOXEA TCSPC analyser," http:// aureatechnology.net/en/products/picoxea.html (06 June 2016).

49. G. N. Chen and Q. Zhu, "Time-resolved optical measurements with spread spectrum excitation," Opt. Lett. (20), 1806-1808 (2002).

50. G. N. Chen and Q. Zhu, "Time-resolved diffusive optical imaging using pseudorandom bit sequences," Opt. Express 11(25), 3445-3454 (2003).

51. D. Borycki et al., "Interferometric near-infrared spectroscopy (iNIRS) for determination of optical and dynamical properties of turbid media," Opt. Express 24(1), 329-354 (2016).

52. J. Fenn and M. Raskino, Mastering the Hype Cycle-How to Choose the Right Innovation at the Right Time, Harvard Business Press, Boston, Massachusetts (2008).

53. A. T. Eggebrecht et al., "Mapping distributed brain function and networks with diffuse optical tomography," Nat. Photonics 8(6), 448-454 (2014).

54. F. Jobsis, "Noninvasive, infrared monitoring of cerebral and myocardial oxygen sufficiency and circulatory parameters," Science 198(4323), 1264-1267 (1977).

55. F. Martelli et al., Light Propagation through Biological Tissue and Other Diffusive Media: Theory, Solutions, and Software, SPIE Press, Bellingham, Washington (2009).

56. Z. Deng et al., "Determination of continuous complex refractive index dispersion of biotissue based on internal reflection," J. Biomed. Opt. 21(1), 015003 (2016).

57. P. Taroni et al., "Noninvasive assessment of breast cancer risk using time-resolved diffuse optical spectroscopy," J. Biomed. Opt. 15(6), 060501 (2010).

58. D. Golovko et al., "Optical imaging of rheumatoid arthritis," Int. J. Clin. Rheumtol. 6(1), 67-75 (2011).

59. J. C. Hebden et al., "Imaging changes in blood volume and oxygenation in the newborn infant brain using three-dimensional optical tomography," Phys. Med. Biol. 49(7), 1117-1130 (2004).

60. A. P. Gibson et al., "Imaging changes in blood volume and oxygenation in the newborn infant brain using three-dimensional optical tomography," Neuroimage 30(2), 521-528 (2006).

61. S. Del Bianco, F. Martelli, and G. Zaccanti, "Penetration depth of light re-emitted by a diffusive medium: theoretical and experimental investigation," Phys. Med. Biol. 47(23), 4131-4144 (2002).

62. J. Steinbrink et al., "Determining changes in NIR absorption using a layered model of the human head," Phys. Med. Biol. 46(3), 879-896 (2001).

63. H. Wabnitz et al., "Performance assessment of time-domain optical brain imagers, part 2: nEUROPt protocol," J. Biomed. Opt. 19(8), 086010 (2014).

64. D. Contini et al., "Novel method for depth-resolved brain functional imaging by time-domain NIRS," Proc. SPIE 6629, 662908 (2007).

65. J. Selb, D. K. Joseph, and D. A. Boas, "Time-gated optical system for depth-resolved functional brain imaging," J. Biomed. Opt. 11(4), 044008 (2006).

66. L. Zucchelli et al., "Method for the discrimination of superficial and deep absorption variations by time domain fNIRS," Biomed. Opt. Express 4(12), 2893-2910 (2013).

67. L. Spinelli et al., "Time-resolved optical stratigraphy in turbid media," Proc. SPIE 7371, 73710A (2009).

68. A. Torricelli et al., "Time-resolved reflectance at null source-detector separation: improving contrast and resolution in diffuse optical imaging," Phys. Rev. Lett. 95(7), 078101 (2005).
69. A. Pifferi et al., "Time-resolved diffuse reflectance at null source-detector separation using a fast gated single-photon avalanche diode," Phys. Rev. Lett. 100(13), 138101 (2008).

70. E. Alerstam et al., "Single-fiber diffuse optical time-of-flight spectroscopy," Opt. Lett. 37(14), 2877-2879 (2012).

71. M. Mazurenka et al., "Non-contact in vivo diffuse optical imaging using a time-gated scanning system," Biomed. Opt. Express 4(10), 2257-2268 (2013).

72. A. Puszka et al., "Spatial resolution in depth for time-resolved diffuse optical tomography using short source-detector separations," Biomed. Opt. Express 6(1), 1-10 (2014).

73. A. Dalla Mora et al., "Towards next-generation time-domain diffuse optics for extreme depth penetration and sensitivity," Biomed. Opt. Express 6(5), 1749-1760 (2015).

74. V. A. Markel and J. C. Schotland, "Effects of sampling and limited data in optical tomography," Appl. Phys. Lett. 81(7), 1180-1182 (2002).

75. B. R. White and J. P. Culver, "Quantitative evaluation of high-density diffuse optical tomography: in vivo resolution and mapping performance," J. Biomed. Opt. 15(2), 026006 (2010).

76. F. Scholkmann et al., "A review on continuous wave functional nearinfrared spectroscopy and imaging instrumentation and methodology," Neuroimage 85(1), 6-27 (2014).

77. A. Bassi et al., "Time-resolved spectrophotometer for turbid media based on supercontinuum generation in a photonic crystal fiber," Opt. Lett. 29(20), 2405-2407 (2004).

78. F. Bevilacqua et al., "Broadband absorption spectroscopy in turbid media by combined frequency-domain and steady-state methods," Appl. Opt. 39(34), 6498-6507 (2000).

79. R. Re et al., "A compact time-resolved system for near infrared spectroscopy based on wavelength space multiplexing," Rev. Sci. Instrum. 81(11), 113101 (2010).

80. H. Wabnitz et al., "A time-domain NIR brain imager applied in functional stimulation experiments," Proc. SPIE 5859, 58590H (2005).

81. D. Contini et al., "Multichannel time-resolved tissue oximeter for functional imaging of the brain," IEEE Trans. Instrum. Meas. 55(1), 85-90 (2006).

82. M. Kacprzak et al., "Time-resolved optical imager for assessment of cerebral oxygenation," J. Biomed. Opt. 12(3), 034019 (2007).

83. A. Pifferi et al., "Fully automated time domain spectrometer for the absorption and scattering characterization of diffusive media," Rev. Sci. Instrum. 78(5), 053103 (2007).

84. Fianium UK Ltd., "WhiteLAse SC sources and accessories" http://www .fianium.com/supercontinuum.htm (06 June 2016).

85. NKT Photonics, "SuperK supercontinuum sources," http://www. nktphotonics.com/supercontinuum_sources (06 June 2016).

86. A. Bassi et al., "Portable, large-bandwidth time-resolved system for diffuse optical spectroscopy," Opt. Express 15(22), 14482-14487 (2007).

87. J. Selb et al., "Functional brain imaging with a supercontinuum timedomain NIRS system," Proc. SPIE 8578, 857807 (2013).

88. M. Diop et al., "Time-resolved near-infrared technique for bedside monitoring of absolute cerebral blood flow," Proc. SPIE 7555, 75550Z (2010).

89. R. Erdmann et al., New Compact and Flexible Picosecond Laser System for Multi-Wavelength Time-Resolved Tissue Spectroscopy, ABSTRACT JW3A.1, OSA Biomedical Optics, Fort Lauderdale, Florida (2016).

90. Becker-Hickl GmbH, "BDS-MM Series," http://www.becker-hickl.de/ bds-mm.htm (06 June 2016).

91. U. M. Weigel et al., The BabyLux Project-An Optical Neuro-Monitor of Cerebral Oxygen Metabolism and Blood Flow for Neonatology, ABSTRACT JM3A.27, OSA Biomedical Optics, Fort Lauderdale, Florida (2016).

92. H. Moench et al., "High-power VCSEL systems and applications," Proc. SPIE 9348, 93480W (2015).

93. M. Born and E. Wolf, Principles of Optics: Electromagnetic Theory of Propagation, Interference and Diffraction of Light, Cambridge University Press, Cambridge, United Kingdom (1997).

94. A. Liebert et al., "Fiber dispersion in time domain measurements compromising the accuracy of determination of optical properties of strongly scattering media," J. Biomed. Opt. 8(3), 512-516 (2003).

95. FiberFin (2016), http://www.fiberfin.com/index.php/products/fiberand-cable/graded-index-pof.html.

96. M. Rehberger et al., "Fiber-based hybrid probe for non-invasive cerebral monitoring in neonatology," Proc. SPIE 9538, 95381J (2015). 
97. M. Mazurenka et al., "Non-contact time-resolved diffuse reflectance imaging at null source-detector separation," Opt. Express 20(1), 283-290 (2012).

98. D. Milej et al., "Estimation of light detection efficiency for different light guides used in time-resolved near-infrared spectroscopy," Biocybernetics Biomed. Eng. 35(4), 227-231 (2015).

99. W. Becker, Advanced Time-Correlated Single-Photon Counting, Springer, Berlin (2005).

100. S. Cova et al., "Avalanche photodiodes and quenching circuits for single-photon detection," Appl. Opt. 35(12), 1956-1976 (1996).

101. R. H. Hadfield, "Single-photon detectors for optical quantum information applications," Nature Photonics 3(12), 696-705 (2009).

102. F. Villa et al., "CMOS SPADs with up to $500 \mu \mathrm{m}$ diameter and $55 \%$ detection efficiency at $420 \mathrm{~nm}$, , J. Mod. Opt. 61(2), 102-115 (2014).

103. H. Wabnitz et al., "Performance assessment of time-domain optical brain imagers, part 1: basic instrumental performance protocol," J. Biomed. Opt. 19(8), 086010 (2014).

104. A. Pifferi et al., "Performance assessment of photon migration instruments: the MEDPHOT protocol," Appl. Opt. 44(11), 2104-2114 (2005).

105. S. Konugolu Venkata Sekar et al., "Broadband $(600-1350 \mathrm{~nm})$ time resolved diffuse optical spectrometer for clinical use," IEEE J. Sel. Top. Quantum Electron. 22(3), 7100609 (2016).

106. A. Puszka et al., "Time-resolved diffuse optical tomography using fastgated single-photon avalanche diodes," Biomed. Opt. Express 4(8), 1351-1365 (2013).

107. L. Di Sieno et al., "Towards non-invasive assessment of flap viability with time-resolved diffuse optical tomography: a pre-clinical test on rats," J. Biomed. Opt. 21(2), 025004 (2016).

108. P. Zipfl, H. Schneckenburger, and L. Schoch, "Fast photomultiplier tube gating technique for time-resolved fluorescence measurements," Proc. SPIE 3600, 158-164 (1999).

109. J. S. Milnes et al., "Ultra-high speed photomultiplier tubes with nanosecond gating for fusion diagnostics," Rev. Sci. Instrum. 83(10), 10D301 (2012).

110. B. A. Jacoby, D. E. Kotecki, and R. D. Lear, "Direct gating of microchannel plates," IEEE Trans. Nucl. Sci. 30(6), 4624-4627 (1983).

111. J. Selb, D. K. Joseph, and D. A. Boas, "Time-gated optical system for depth-resolved functional brain imaging," J. Biomed. Opt. 11(4), 044008 (2006).

112. P. Sawosz et al., "Application of time-gated CCD camera with image intensifier in contactless detection of absorbing inclusions buried in optically turbid medium which mimics local changes in oxygenation of the brain tissue," Opto-Electron. Rev. 20(4), 309-314 (2012).

113. Hamamatsu Photonics K.K., "Guide to streak cameras," https://www. hamamatsu.com/resources/pdf/sys/SHSS0006E_STREAK.pdf (06 June 2016).

114. A. Dalla Mora et al., "Afterpulse-like noise limits dynamic range in time-gated applications of thin-junction silicon single-photon avalanche diode," Appl. Phys. Lett. 100(24), 241111 (2012).

115. A. Dalla Mora et al., "Single-photon avalanche diode model for circuit simulations," IEEE Photonics Technol. Lett. 19(23), 1922-1924 (2007).

116. A. Dalla Mora et al., "Fast-gated single-photon avalanche diode for wide dynamic range near infrared spectroscopy," IEEE J. Sel. Top. Quantum Electron. 16(4), 1023-1030 (2010).

117. G. Boso et al., "Fast-gating of single-photon avalanche diodes with 200 ps transitions and 30 ps timing jitter," Sens. Actuators A Phys. 191(1), 61-67 (2013).

118. M. Buttafava et al., "Time-gated single-photon detection module with 110 ps transition time and up to $80 \mathrm{MHz}$ repetition rate," Rev. Sci. Instrum. 85(8), 083114 (2014).

119. A. Dalla Mora et al., "Memory effect in silicon time-gated singlephoton avalanche diodes," J. Appl. Phys. 117(11), 114501 (2015).

120. A. Tosi et al., "Fast-gated single-photon counting technique widens dynamic range and speeds up acquisition time in time-resolved measurements," Opt. Express 19(11), 10735-10746 (2011).

121. F. Zappa et al., "SPICE modeling of single photon avalanche diodes," Sens. Actuators A: Phys. 153(2), 197-204 (2009).

122. D. S. Bethune and W. P. Risk, "An autocompensating fiber-optic quantum cryptography system based on polarization splitting of light," IEEE J. Quantum Electron. 36(3), 340-347 (2000).
123. D. S. Bethune et al., "System for gated detection of optical pulses containing a small number of photons using an avalanche photodiode," U.S. Patent 6,218,657 (2001).

124. A. Tomita and K. Nakamura, "Balanced gated-mode photon detector for quantum-bit discrimination at $1550 \mathrm{~nm}$," Opt. Lett. 27(20), 18271829 (2002).

125. A. Yoshizawa, R. Kaji, and H. Tsuchida, "Gated-mode single-photon detection at $1550 \mathrm{~nm}$ by discharge pulse counting," Appl. Phys. Lett. 84(18), 3606-3608 (2004).

126. C. Zhou, G. Wu, and H.P. Zeng, "Multigate single-photon detection and timing discrimination with an InGaAs/InP avalanche photodiode," Appl. Opt. 45(8), 1773-1776 (2006).

127. Z. Wei et al., "An integral gated mode single photon detector at telecom wavelengths," J. Phys. D: Appl. Phys. 40(22), 6922-6925 (2007).

128. Z. L. Yuan et al., "High speed single photon detection in the near infrared," Appl. Phys. Lett. 91(4), 041114 (2007).

129. S. Cova, G. Ripamonti, and A. Lacaita, "Avalanche semiconductor detector for single optical photons with a time resolution of $60 \mathrm{ps,"}$ Nucl. Instrum. Methods A 253(3), 482-487 (1987).

130. D. Contini, "Effects of time-gated detection in diffuse optical imaging at short source-detector separation," J. Phys. D: Appl. Phys. 48(4), 045401 (2015).

131. E. Charbon, "Towards large scale CMOS single-photon detector arrays for lab-on-chip applications," J. Phys. D: Appl. Phys. 41(9), 094010 (2008).

132. F. Villa et al., "CMOS imager with 1024 SPADs and TDCS for singlephoton timing and 3-D time-of-flight," IEEE J. Sel. Top. Quantum Electron. 20(6), 3804810 (2014).

133. B. Dolgoshein et al., "Status report on silicon photomultiplier development and its applications," Nucl. Instrum. Methods A 563(2), 368-376 (2006).

134. D. Renker, "New trends on photodetectors," Nucl. Instrum. Methods A 571(1-2), 1-6 (2007).

135. S. Cova, "Trapping phenomena in avalanche photodiodes on nanosecond scale," IEEE Electron Device Lett. 12(12), 685-687 (1991).

136. P. Eraerds et al., "SiPM for fast photon-counting and multiphoton detection," Opt. Express. 15(22), 14539-14549 (2007).

137. M. Akiba, K. Inagaki, and K. Tsujino, "Photon number resolving SiPM detector with $1 \mathrm{GHz}$ count rate," Opt. Express 20(3), 2779-2788 (2012).

138. P. Buzhan et al., "Silicon photomultiplier and its possible applications," Nucl. Instrum. Methods A 504(1-3), 48-52 (2003).

139. S. Vinogradov, "Analytical model of SiPM time resolution and order statistics with crosstalk," Nucl. Instrum. Methods A 787(1), 229-233 (2015).

140. G. Galetta et al., "Time and charge characterization of Hamamatsu photonics silicon photomultipliers," EPJ Web Conf. 66(1), 11028 (2014).

141. F. Acerbi et al., "Characterization of single-photon time resolution: from single SPAD to silicon photomultiplier," IEEE Trans. Nucl. Sci. 61(5), 2678-2686 (2014).

142. C. Jackson et al., "Ultra-low noise and exceptional uniformity of SensL C-series SiPM sensors," Proc. SPIE 9359, 93591C (2015).

143. P. Berard et al., "Performance measurement for a new low dark count UV-SiPM," in Nuclear Science Symp. and Medical Imaging Conf. (NSS/MIC), pp. 544-547 (2011).

144. R. Zimmermann et al., "Silicon photomultipliers for improved detection of low light levels in miniature near-infrared spectroscopy instruments," Biomed. Opt. Express 4(5), 659-666 (2013).

145. D. Sanfilippo et al., "Design and development of a fNIRS system prototype based on SiPM detectors," Proc. SPIE 8990, 899016 (2014).

146. A. Dalla Mora et al., "Fast silicon photomultiplier improves signal harvesting and reduces complexity in time-domain diffuse optics," Opt. Express 23(11), 13937-13946 (2015).

147. E. Martinenghi et al., "Spectrally resolved single-photon timing of silicon photomultipliers for time-domain diffuse spectroscopy," IEEE Photonics J. 7(4), 1-12 (2015).

148. S. Konugolu Venkata Sekar et al., "Time-resolved diffused optical characterization of key tissue constituents of human bony prominence locations," Proc. SPIE 9538, 95380X (2015).

149. S. Fujisaka et al., "A clinical tissue oximeter using NIR time-resolved spectroscopy," in Proc. 43rd Annual Meeting of the Int. Society on Oxygen Transport to Tissue (ISOTT), C. E. Elwell, T. S. Leung, and D. K. Harrison, Eds., Vol. 876, pp. 427-234 (2016). 
150. F. Villa et al., "SPICE electrical models and simulations of silicon photomultipliers," IEEE Trans. Nucl. Sci. 62(5), 1950-1960 (2015).

151. F. Villa et al., "Analog SiPM in planar CMOS technology," in Proc. of Solid State Device Research Conf. (ESSDERC), Venice, Italy, pp. 294297, IEEE, New York, New Jersey (2014).

152. T. Svensson et al., "Near-infrared photon time-of-flight spectroscopy of turbid materials up to $1400 \mathrm{~nm}$," Rev. Sci. Instrum. 80(6), 063105 (2009).

153. A. Tosi et al., "Single-photon avalanche diodes for the near-infrared range: detector and circuit issues," J. Mod. Opt. 56(2-3), 299-308 (2009).

154. A. Tosi et al., "Active area uniformity of $\mathrm{InGaAs} / \mathrm{InP}$ single-photon avalanche diodes," IEEE Photonics J. 3(1), 31-41 (2011).

155. I. Bargigia et al., "Time-resolved diffuse optical spectroscopy up to $1700 \mathrm{~nm}$ by means of a time-gated InGaAs/InP single-photon avalanche diode," Appl. Spectrosc. 66(8), 944-950 (2012).

156. I. Bargigia et al., "Time-domain diffuse optical spectroscopy up to $1700 \mathrm{~nm}$ using an InGaAs/InP single-photon avalanche diode," Proc. SPIE 7895, 78950C (2011).

157. I. Bargigia et al., "In-vivo optical spectroscopy in the time-domain beyond 1100 nm," Proc. SPIE 8799, 879902 (2013).

158. X. Jiang et al., "Negative feedback avalanche diodes for near-infrared single photon detection," Proc. SPIE 7320, 732011 (2009).

159. J. Zhang et al., "Advances in InGaAs/InP single-photon detector systems for quantum communication," Light: Sci. Appl. 4(11), e286 (2015).

160. X. Jiang et al., "Shortwave infrared negative feedback avalanche diodes and solid-state photomultipliers," Opt. Eng. 53(8), 081908 (2014).

161. D. V. O'Connor and D. Phillips, Time Correlated Single Photon Counting, Academic Press, London (1984).

162. W. Becker, (Ed.), Advanced Time-Correlated Single Photon Counting Applications, Springer, Berlin (2015).

163. J. Kalisz, "Review of methods for time interval measurements with picosecond resolution," Metrologia 41(1), 17-32 (2004).

164. Becker-Hickl GmbH, "TCSPC-Modules," (2016) http://www. becker-hickl.de/products.htm\#TCSPC\%20MODULES (06 June 2016).

165. Picoquant GmbH, "TCSPC and time tagging electronics," http://www. picoquant.com/products/category/tcspc-and-time-tagging-modules (06 June 2016).

166. B. Markovic et al., " 10 ps resolution, $160 \mathrm{~ns}$ full scale range and less than $1.5 \%$ differential non-linearity time-to-digital converter module for high performance timing measurements," Rev. Sci. Instrum. 83(7), 074703 (2012).

167. B. Markovic et al., "A high-linearity, 17 ps precision time-to-digital converter based on a single-stage Vernier delay loop fine interpolation," IEEE Trans. Circuits Syst. I-Regul. Paper 60(3), 557-569 (2013).

168. F. Villa et al., "SPAD smart pixel for time-of-flight and time-correlated single-photon counting measurements," IEEE Photonics J. 4(3), 795804 (2012).

169. C. Veerappan et al., "A $160 \times 128$ single-photon image sensor with onpixel 55 ps $10 \mathrm{~b}$ time-to-digital converter," in IEEE Int. Solid-State Circuits Conf. (ISSCC), Digest of Technical Papers, pp. 312-314 (2011).

170. M. Gersbach et al., "A time-resolved, low-noise single-photon image sensor fabricated in deep-submicron CMOS technology," IEEE J. Solid-State Circuit 47(6), 1394-1407 (2012).

171. S. Jahromi et al., "A single chip laser radar receiver with a $9 \times 9$ SPAD detector array and a 10-channel TDC," in European Solid-State Circuits Conf., pp. 364-367, IEEE, New York, New York (2015).

172. E. Charbon, "Single-photon imaging in complementary metal oxide semiconductor processes," Phil. Trans. R. Soc. A 372, 20130100 (2014).

173. J. Mata Pavia, M. Wolf, and E. Charbon, "Single-photon avalanche diode imagers applied to near-infrared imaging," IEEE J. Sel. Top. Quantum Electron. 20(6), 291-298 (2014).

174. Philips Digital Photon Counting, "Technology," http://www. digitalphotoncounting.com/ (06 June 2016).

175. P. B. Coates, "The correction for photon 'pile-up' in the measurement of radiative lifetimes," J. Phys. E: Sci. Instrum. 1(8), 878 (1968).

176. D. Tyndall et al., "A high-throughput time-resolved mini-silicon photomultiplier with embedded fluorescence lifetime estimation in $0.13 \mu \mathrm{m}$ CMOS," IEEE Trans. Biomed. Circuits Syst. 6(6), 562-570 (2012).
177. S. Mandai and E. Charbon, "Multi-channel digital SiPMs: concept, analysis and implementation," in IEEE Nuclear Science Symp. and Medical Imaging Conf. (NSS/MIC), pp. 1840-1844, IEEE, New York, New York (2012).

178. N. A. W. Dutton et al. "11.5 A time-correlated single-photon-counting sensor with 14GS/S histogramming time-to-digital converter," in IEEE Int. Solid-State Circuits Conf., pp. 1-3, IEEE, New York, New York (2015).

179. N. Krstajić et al., " 0.5 billion events per second time correlated single photon counting using CMOS SPAD arrays," Opt. Lett. 40(18), 43054308 (2015).

180. G. Boso et al., "High-throughput gated photon counter with two detection windows programmable down to $70 \mathrm{ps}$ width," Rev. Sci. Instrum. 85(1), 013107 (2014).

181. L. Sieno et al., "Diffuse optics using a dual window fast-gated counter," Appl. Opt. 53(31), 7394-7401 (2014).

182. L. Spinelli et al., "In-vivo swine myocardial tissue characterization and monitoring during open chest surgery by time-resolved diffuse nearinfrared spectroscopy," Proc. SPIE 7883, 78833D (2011).

183. G. Quarto et al. "Time-resolved optical spectroscopy of the chest: is it possible to probe the lung?," Proc. SPIE 8799, 87990Q (2013).

184. N. Ramanujam et al., "Photon migration through fetal head in utero using continuous wave, near infrared spectroscopy: clinical and experimental model studies," J. Biomed. Opt. 5(2), 173-84 (2000).

185. R. Choe et al., "Transabdominal near infrared oximetry of hypoxic stress in fetal sheep brain in utero," Proc. Natl. Acad. Sci. U. S. A. 100(22), 12950-12954 (2003).

186. K. Uludag and A. Roebroek, "General overview on the merits of multimodal neuroimaging data fusion," Neuroimage 102(1), 3-10 (2014).

187. R. H. Wilson et al., "Review of short-wave infrared spectroscopy and imaging methods for biological tissue characterization," J. Biomed. Opt. 20(3), 030901 (2015).

188. G. Quarto et al., "Estimate of tissue composition in malignant and benign breast lesions by time-domain optical mammography," Biomed. Opt. Express 5(10), 3684-3698 (2014).

189. P. Taroni et al., "Optical identification of subjects at high risk for developing breast cancer," J. Biomed. Opt. 18(6), 060507 (2013).

190. A. Pifferi et al., "Collagen content as a risk factor in breast cancer? A pilot clinical study," Proc. SPIE 9538, 953813 (2015).

191. J. Couzin, "Dissecting a hidden breast cancer risk," Science 309(5741), 1664-1666 (2005).

192. A. Liemert and A. Kienle, "Exact and efficient solution of the radiative transport equation for the semi-infinite medium," Sci. Rep. 3, 2018 (2013).

193. E. Simon, F. Foschum, and A. Kienle, "Hybrid Green's function of the time-dependent radiative transfer equation for anisotropically scattering semi-infinite media," J. Biomed. Opt. 18(1), 015001 (2013).

194. A. Dalla Mora et al., "Towards next generation time-domain diffuse optics devices," Proc. SPIE 9319, 93191J (2015).

195. M. Raheel Bhutta et al., "Single-trial lie detection using a combined fNIRS-polygraph system," Front Psychol. 6, 709 (2015).

196. F. Tian et al., "Functional near-infrared spectroscopy to investigate hemodynamic responses to deception in the prefrontal cortex," Brain Res. 1303, 120-130 (2009).

197. X. S. Hu, K.-S. Hong, and S. S. Ge, "fNIRS-based online deception decoding," J. Neural Eng. 9(2), 026012 (2012).

198. X. P. Ding et al., "Neural correlates of second-order verbal deception: a functional near-infrared spectroscopy (fNIRS) study," Neuroimage 87, 505-514 (2014).

199. L. Sai et al., "Detecting concealed information using functional nearinfrared spectroscopy," Brain Topogr. 27(5), 652-662 (2014).

200. T. Koike, H. C. Tanabe, and N. Sadato, "Hyperscanning neuroimaging technique to reveal the" two-in-one" system in social interactions," Neurosci. Res. 90(1), 25-32 (2015).

201. T. Muehlemann et al., "In vivo functional near-infrared spectroscopy measures mood-modulated cerebral responses to a positive emotional stimulus in sheep," Neuroimage 54(2), 1625-1633 (2011).

202. S. Vögeli et al., "Housing conditions influence cortical and behavioural reactions of sheep in response to videos showing social interactions of different valence," Behav. Brain Res. 284, 69-76 (2015).

203. S. Vögeli et al., "Frontal brain activity and behavioral indicators of affective states are weakly affected by thermal stimuli in sheep living in different housing conditions," Front. Vet. Sci. 2, 9 (2015). 
204. L. Gygax et al., "Dog behavior but not frontal brain reaction changes in repeated positive interactions with a human: a non-invasive pilot study using functional near-infrared spectroscopy (fNIRS)," Behav. Brain Res. 281, 172-176 (2015).

205. European Commission, "EU animal welfare strategy: 2012-2015," http://ec.europa.eu/food/animal/welfare/actionplan/docs/aw_strategy_ 19012012_en (06 June 2016).

206. E. Check Hayden, "Why biomedical superstars are signing on with Google," Nature 526(7574), 484-485 (2015).

207. A. Liebert et al., "Assessment of inflow and washout of indocyanine green in the adult human brain by monitoring of diffuse reflectance at large source-detector separation," J. Biomed. Opt. 16(4), 046011 (2011).

Antonio Pifferi is a full professor in the Department of Physics, Politecnico di Milano (Italy). He received his MS degree in nuclear engineering in 1991 from Politecnico di Milano and the PhD in physics in 1995 from Politecnico di Torino. His research focuses on the development of new laser techniques and instrumentation for diagnosis, and on the study of light propagation in diffusive media, with applications to optical biopsy, optical mammography, and functional brain imaging.

Davide Contini is an associate professor in the Department of Physics, Politecnico di Milano (Italy). He received his MS degree in electronic engineering and the PhD degree in physics from Politecnico di Milano in 2004 and 2007, respectively. He is the author of more than 100 papers in international peer-reviewed journals and conference proceedings. His research activity is focused on time-resolved spectroscopy of highly diffusive media for applications in biology and medicine.

Alberto Dalla Mora has been an assistant professor in the Department of Physics, Politecnico di Milano (Italy), since 2011. He graduated summa cum laude in electronics engineering from Politecnico di Milano in 2006, and he received the PhD degree summa cum laude in information and communication technology from the same university in 2010 . He authored more than 30 papers in international peer-reviewed journals. Currently, his research interests include timeresolved diffuse spectroscopy instrumentation and applications for biomedical diagnosis.

Andrea Farina received his MS in electronics engineering in 2005 and the PhD in physics in 2009 from Politecnico di Milano (Italy). Since 2009, he has been a postdoc in the Department of Physics at Politecnico di Milano. In 2011, he became a researcher for the Italian Research National Council at the Institute of Photonics and Nanotechnologies. His research interests are devoted to the study of photon migration using time-resolved and structured light for imaging and spectroscopy.

Lorenzo Spinelli received his MS and PhD degrees in physics from the University of Milan, Italy, in 1994 and 1999, respectively. Since 1999 , he has been a postdoc in the Department of Physics at the University of Milan. In 2001, he became a researcher for the Italian Research National Council at the Institute of Photonics and Nanotechnologies. His research interests are devoted to the study of photon migration in turbid media for optical biopsy and imaging.

Alessandro Torricelli is an associate professor in the Department of Physics, Politecnico di Milano (Italy). He received his MS degree in electronic engineering from Politecnico di Milano in 1994 and his PhD degree in physics from Politecnico di Torino in 1999. He is the author of more than 100 papers in international peer-reviewed journals. His current research interests include photon migration in diffusive media, functional near infrared spectroscopy, and noninvasive diffuse spectroscopy with time domain systems. 